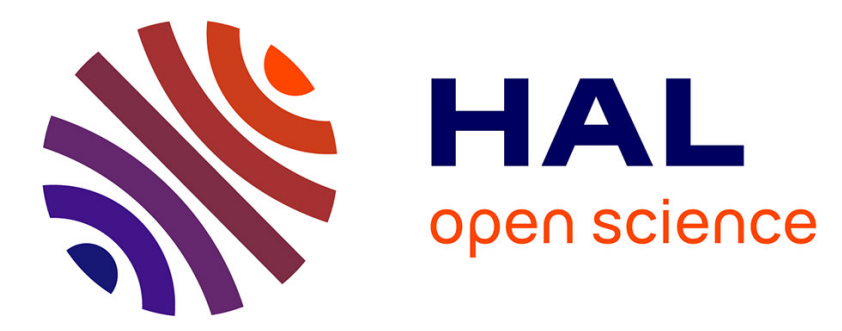

\title{
Spectral gap properties and limit theorems for some random walks and dynamical systems \\ Yves Guivarc'H
}

\section{To cite this version:}

Yves Guivarc'H. Spectral gap properties and limit theorems for some random walks and dynamical systems. D. Dolgopyat, Y. Pesin, M. Pollicott and L. Stoyanov. Hyperbolic Dynamics, Fluctuations and Large Deviations, 89, American Mathematical Society, pp.279-310, 2015, Proceedings of Symposia in Pure Mathematics, 978-1-4704-1112-1. hal-01022414

\section{HAL Id: hal-01022414 https://hal.science/hal-01022414}

Submitted on 10 Jul 2014

HAL is a multi-disciplinary open access archive for the deposit and dissemination of scientific research documents, whether they are published or not. The documents may come from teaching and research institutions in France or abroad, or from public or private research centers.
L'archive ouverte pluridisciplinaire HAL, est destinée au dépôt et à la diffusion de documents scientifiques de niveau recherche, publiés ou non, émanant des établissements d'enseignement et de recherche français ou étrangers, des laboratoires publics ou privés. 


\title{
Spectral gap properties and limit theorems for some random walks and dynamical systems.
}

\author{
Yves Guivarc'h
}

\begin{abstract}
We give a description of some limit theorems and the corresponding proofs for various transfer operators. Our examples are closely related with random walks on homogeneous spaces. The results are obtained using spectral gap methods in Hölder spaces or Hilbert spaces. We describe also their geometrical setting and the basic corresponding properties. In particular we focus on precise large deviations for products of random matrices, Fréchet's law for affine random walks and local limit theorems for Euclidean motion groups or nilmanifolds.
\end{abstract}

\section{Introduction}

In the proofs of limit theorems for dynamical systems with hyperbolic properties, the use of transfer operators plays an important role. For transformations with expanding properties this amount to the use of spectral gap properties of Perron-Frobenius operators (see for example [7], [20], [37], [46]). For Anosov systems and their extensions, the relevant transfer operators are obtained through the use of a Markov partition which allows to go over to a symbolic system (see [5], [48]) and to prove limit theorems (see [25]). In the context of random walks on homogeneous spaces, spectral gap properties on Hölder spaces ([17], [25], [28]), $\mathbb{L}^{2}$-spaces (see [1], [3], [10], [15]), or Sobolev spaces (see [9], [11]) are valid also. Hence we are led to study a general class of Markov chains on a metric space $(X, d)$ associated with semigroup actions on $X$.

Let $m$ be a probability on the Borel subsets of the complete and separable metric space $(X, d), S$ a Polish semigroup acting measurably on $X$ by $x \rightarrow g . x(x \in X, g \in S)$. Let $p(x, g)$ be a non negative measurable function on $(X, S)$ with $\int p(x, g) d \mu(g)=1$ for any $x \in X$, where $\mu \in M^{1}(S)$ is a probability on $S$. We consider the Markov operator $Q$ defined on bounded measurable functions by $Q \varphi(x)=\int \varphi(g \cdot x) p(x, g) d \mu(g)$. The special case where $p(x, g)=1$ is often considered in the framework of the so-called "Iterated Random Functions" (see [32] for example). In various situations like the study of expanding transformations (see [7]) or large deviations for products of random matrices (see below) it is natural to allow $p(x, g)$ to depend on $(x, g) \in X \times S$. We will assume that $m$ is $Q$-invariant i.e $Q m=m$ where $Q m(\varphi)=m(Q \varphi)$. We denote by $\Omega$ the product space $\Omega=S^{\mathbb{N}}$ and for $\omega=\left(g_{1}, g_{2}, \cdots\right) \in \Omega, n \in \mathbb{N}$, we write $s_{n}=s_{n}(\omega)=g_{n} \cdots g_{1} \in S$, $p_{n}(x, \omega)=\prod_{k=1}^{n} p\left(s_{k-1} \cdot x, g_{k}\right)$. We define the probability $\mathbb{P}_{x}$ on $\Omega$ as the projective limit of the system of probabilities $p_{n}(x,.) \mu^{\otimes n}$ on the spaces $\Omega_{n}=S \times \cdots \times S$ ( $n$ times) and we write $\mathbb{P}=\int \mathbb{P}_{x} d m(x)$. The corresponding expectation with respect to $\mathbb{P}_{x}($ resp. $\mathbb{P})$ will be denoted by $\mathbb{E}_{x}($ resp. $\mathbb{E})$. In particular, we have $Q^{n} \varphi(x)=\mathbb{E}_{x}\left(\varphi \circ s_{n}\right)$.

Then the $Q$-invariance of $m$ implies that $\mathbb{P}$ is invariant under the shift $\theta$ on $\Omega$. If $m$ is 
$Q$-ergodic, then the dynamical system $(\Omega, \theta, \mathbb{P})$ is also ergodic. We will be interested in limit theorems for the Birkhoff sum $S_{n}(x, \omega)=\sum_{1}^{n} f\left(s_{k-1} . x, g_{k}\right)$ where $f(x, g)$ is a given real valued Borel function on $X \times S$. It is natural to consider the associated "Markov walk" on $X \times \mathbb{R}$ given by $(x, t) \rightarrow\left(g_{1} \cdot x, t+f\left(x, g_{1}\right)\right)$, its kernel denoted by $\dot{Q}$, leaves invariant the measure $m \otimes \ell$ where $\ell$ is Lebesgue measure on $\mathbb{R}$ and $\dot{Q}$ commutes with the translations on $X \times \mathbb{R}$ given by $(x, t) \rightarrow\left(x, t+t^{\prime}\right)$. The iterated transitions $(x, t) \rightarrow\left(s_{n} . x, t+S_{n}(x,).\right)$ are given by the iterated kernel $\dot{Q}^{n}$. Here we restrict mainly to the problem of the local limit theorem i.e to the asymptotics of $\mathbb{E}\left[1_{I}\left(S_{n}(x,).\right]\right.$ where $I$ is an interval of the line. The Fourier transform of the random variable $S_{n}$, i.e $\mathbb{E}_{x}\left(e^{i t S_{n}(x, \cdot)}\right.$ where $t \in \mathbb{R}$, can be calculated with the "Fourier operators" $Q^{i t}$ given on bounded functions on $X$ by $Q^{i t} \varphi(x)=$ $\int \varphi(g . x) p(x, g) e^{i t f(x, g)} d \mu(g)$ and, with abuse of notation, we write $Q^{i t} \varphi(x)=Q\left(e^{i t f} \varphi\right)$. In particular we have : $\mathbb{E}_{x}\left(e^{i t S_{n}(x, .)} \varphi\left(s_{n} . x\right)\right)=\left(Q^{i t}\right)^{n} \varphi(x)$.

The spectral methods developed below in specific examples are also useful for proving precise forms of other limit theorems like central limit thorem with remainder (see [20], [42], [46]), large deviations, renewal theorem (see [25] for example), but also for convergence to stable laws $([28])$ and extreme value theory ([33]). For recent results on random walks in random medium, closely connected with extreme value theory and stable laws on the affine group see [13].

In section 1 we recall basic spectral results and we show their connexion with the local limit theorem. In section 2 we describe results for products of random matrices which are used in sections 3,4 . In section 3 we apply the results of sections 1,2 to some specific situations ; we get local limit theorems on motion groups and on nilmanifolds, as well as large deviations for products of random matrices. In section 4 we consider affine random walks on $\mathbb{R}^{d}$ and we apply the above spectral results to get an analogue of Fréchet's law, in particular. Sections 3,4 and the end of section 2 corresponds to recent results (see [31], [33]). For the beginning of section 2, see the surveys [26], [30] and the book [4].

We thank B. Bekka, J.-P Conze, G.-A Margulis for important remarks and the referee for very useful suggestions.

\section{Spectral gap properties of Markov operators and the local limit theorem.}

We will show the local limit theorem in various geometric situations and we begin by recalling a useful analytic setting first considered in [12] and fully developed in [35]. We assume that the metric space $(X, d)$ is compact and we write $C(X)$ for the space of continuous functions ; if $\varphi \in C(X)$ we denote for $\varepsilon>0|\varphi|_{\infty}=\sup _{x}|\varphi(x)|,[\varphi]_{\varepsilon}=\sup _{x, y} \frac{|\varphi(x)-\varphi(y)|}{d^{\varepsilon}(x, y)}$ and we write $H^{\varepsilon}(X) \subset C(X)$ for the space of $\varepsilon$-Hölder functions i.e $\varphi \in H^{\varepsilon}(X)$ if and only if $[\varphi]_{\varepsilon}<\infty$. The space $H^{\varepsilon}(X)$ is normed by $\|\varphi\|=|\varphi|_{\infty}+[\varphi]_{\varepsilon}$ and then it becomes a 
Banach space. We will say that a bounded operator $Q$ on $C(X)$ satisfies condition (D-F) if

a) The powers $Q^{n}$ of $Q$ acting on $C(X)$ are uniformly bounded in norm ||$_{\infty}$.

b) There exists numbers $\rho \in\left[0,1\left[, r \in \mathbb{N}, C \geq 0\right.\right.$ such that for any $\varphi \in H^{\varepsilon}(X)$ $\left[Q^{r} \varphi\right]_{\varepsilon} \leq \rho[\varphi]_{\varepsilon}+C|\varphi|_{\infty}$

The functional inequality in b) will we called (D-F) inequality.

Then we have the

Theorem 1.1 Assume that $(X, d)$ is compact and $Q$ satisfies condition $(D-F)$ on $H^{\varepsilon}(X)$. Then $Q$ has only finitely many unimodular spectral values and they are isolated in the spectrum of $Q$. The corresponding characteristic subspaces have finite dimension and are equal to the corresponding eigenspaces.

For more general and more precise statements see [12], [34], [35].

If $Q$ is a Markov operator on $X$, hence in particular $Q 1=1$, we say that $Q$ is irreducible if the equation $Q \varphi=e^{i \theta} \varphi(\theta \in \mathbb{R})$ implies $e^{i \theta}=1, \varphi=$ const. If the Markov operator $Q$ on $X$ is irreducible and satisfies condition (D-F) then $m$ is the unique $Q$-invariant measure and the projection $m \otimes 1$ on $\mathbb{C} 1$ satisfies $\lim _{n \rightarrow \infty}\left\|Q^{n}-m \otimes 1\right\|=0$. More precisely, we have the spectral decomposition $Q=m \otimes 1+R$ where $R(m \otimes 1)=(m \otimes 1) R=0$ and the spectral radius of $R$ is less than 1 . We write $H_{0}^{\varepsilon}(X)=\left\{\varphi \in H^{\varepsilon}(X) ; m(\varphi)=0\right\}$ and we observe that $H^{\varepsilon}(X)=\mathbb{C} 1 \oplus H_{0}^{\varepsilon}(X)$. Under condition D-F, $Q$-ergodicity of $m$ is equivalent to simplicity of the eigenvalue 1. Also, the $\operatorname{support} \operatorname{supp}(m)$ of $m$ is the smallest closed $Q$-invariant subset of $X$. In general it is smaller than $X$, but we can always restrict $Q$ to $\operatorname{supp}(m)$. For the operator $Q^{i t}$ we have $\left[Q^{i t} \varphi\right]_{\varepsilon} \leq \rho[\varphi]_{\varepsilon}+C_{t}|\varphi|_{\infty}$ where

$\rho=\sup _{x, y} \int p(x, g) \frac{d^{\varepsilon}(g \cdot x, g \cdot y)}{d^{\varepsilon}(x, y)} d \mu(g)$
$C_{t}=\sup _{x, y} \int \frac{\left|e^{i t f(x, g)} p(x, g)-e^{i t f(y, g)} p(y, g)\right|}{d^{\varepsilon}(x, y)} d \mu(g)$.

The corresponding quantities for $\left(Q^{i t}\right)^{n}$ are conveniently expressed with $\mathbb{E}_{x}$, in particular $\rho$ is replaced by :

$\rho(n)=\sup _{x, y}\left|\mathbb{E}_{x}\left(\frac{d^{\varepsilon}\left(s_{n} \cdot x, s_{n} \cdot y\right)}{d^{\varepsilon}(x, y)}\right)\right|$.

In particular if the functions $x \rightarrow p(x, g), x \rightarrow f(x, g)$ are uniformly Hölder then $C_{t}<\infty$. Also, if $\lim _{n \rightarrow \infty} \rho(n)<1$, then condition D-F is satisfied; hence in this case we are able to develop a detailed study of $S_{n}(x, \omega)$.

We assume now that $Q$ is an irreducible Markov operator with satisfies condition D-F and $f(., g)$ is uniformly Hölder. We follow closely [25].

Definition 1.2 We will say that the function $f(x, g)$ on $X \times S$ is non degenerate (resp is aperiodic) if $f$ is not additively (resp multiplicatively) cohomologous to a constant on $\operatorname{supp}(m)$ i.e for any $g \in \operatorname{supp}(\mu), x \in \operatorname{supp}(m)$ and some $u \in H^{\varepsilon}(X), c \in \mathbb{R}$ (resp 
$\left.u \in H^{\varepsilon}(X),|u|=1, t \in \mathbb{R}, \theta \in \mathbb{R}\right)$ we have $f(x, g)=u(g . x)-u(x)+c$ (resp. $e^{i t f(x, g)}=$ $\left.e^{i \theta} u(g . x) u^{-1}(x)\right)$.

We assume that the integral $\int f(x, g) d m(x) d \mu(g)$ is finite and we denote this integral by $\gamma(f)$. The function $v(x)=\int f(x, g) d \mu(g)-\gamma(f)$ satisfies $m(v)=0$, hence the equation $u-Q u=v$ has a unique solution $u$ with $u \in H_{0}^{\varepsilon}(X)$. If $\int f^{2}(x, g) d m(x) d \mu(g)$ is finite we can define

$$
\sigma^{2}=\sigma^{2}(f)=\int(f(x, g)-\gamma(f)+u(x)-u(g \cdot x))^{2} d m(x) d \mu(g)
$$

and non degeneracy of $f$ can be expressed as $\sigma^{2}(f)>0$.

Also we see that for $t$ small, by perturbation theory on $H^{\varepsilon}(X)$, the operator $Q^{i t}$ has a simple dominant eigenvalue $k(f, i t)=k(i t)$ which is isolated in the spectrum of $Q^{i t}$ and satisfies $|k(i t)|=\lim _{n \rightarrow \infty}\left\|\left(Q^{i t}\right)^{n}\right\|^{1 / n}<1$. If $\int f^{2}(x, g) d m(x) d \mu(g)<\infty$ the second derivative of $k(i t)$ at zero exists and $k^{\prime}(f, 0)=i \gamma(f), k^{\prime \prime}(f, 0)=-\left(\sigma^{2}(f)+\gamma^{2}(f)\right)$. The non-degeneracy (resp aperiodicity) of $f$ can be caracterized as follows :

Proposition 1.3 With the above notations, the following conditions are equivalent

a) $f$ is degenerate (resp not aperiodic).

b) For any $t \in \mathbb{R}$ (resp for some $t \neq 0)$, there exists $\theta \in \mathbb{R}$ and $\varphi_{t} \in H^{\varepsilon}(\operatorname{supp}(m))$ such that $Q^{i t} \varphi_{t}=e^{i \theta} \varphi_{t}$ on $\operatorname{supp}(m)$.

The following simple Lemma will be useful in the proof of the local limit theorem below.

Lemma 1.4 With the above notation assume $\int f^{2}(x, g) d m(x) d \mu(g)<\infty, \gamma(f)=0$, and $f$ is non degenerate. Then for any $\varphi \in H^{\varepsilon}(X), t \in \mathbb{R}$, the sequence $\left(Q^{i t / \sqrt{n}}\right)^{n} \varphi$ converges to $e^{-t^{2} \sigma^{2} / 2} m(\varphi)$. There exists $A>0, \varepsilon_{0}>0$ such that for $|t / \sqrt{n}|<\varepsilon_{0}$ the sequence $\left\|\left(\mathbb{Q}^{i t / \sqrt{n}}\right)^{n}\right\|$ is bounded by $2 e^{-A t^{2}}$.

Proof : Since $Q$ is irreducible and satisfies condition (D-F), we have for $t$ small, by perturbation in $H^{\varepsilon}(X), Q^{i t} \varphi=k(i t) m_{t}(\varphi) e_{t}+R(i t) \varphi$ where $m_{t} \otimes e_{t}$ is the projection operator on the line $\mathbb{C} e_{t},\left(m_{t} \otimes e_{t}\right) R(i t)=R(i t)\left(m_{t} \otimes e_{t}\right)=0$, and the spectral radius of $R(i t)$ is less than $|k(i t)|$. Since $\gamma(f)=0$ and $\int f^{2}(x, g) d m(x) d \mu(g)<\infty$ we have $k(i t)=1-\sigma^{2} t^{2} / 2+\circ\left(t^{2}\right)$, hence $|k(i t)|<1$ for $t$ small non zero and $\lim _{n \rightarrow \infty} k^{n}(i t \sqrt{n})=$ $\lim _{n \rightarrow \infty}\left(1-\sigma^{2} t^{2} / 2 n\right)^{n}=e^{-\sigma^{2} t^{2} / 2}$. Also, for some $C \in\left[0,|k(i t)|\left[, C<1\right.\right.$ we have $\left(Q^{i t}\right)^{n}=$ $k^{n}(i t) m_{t} \otimes e_{t}+R^{n}(i t)$ with $\left\|(R(i t))^{n}\right\| \leq C^{n}, \lim _{t \rightarrow 0} m_{t} \otimes e_{t}=m \otimes 1$. This gives the first conclusion. The asymptotic expansion of $k(i t)$ gives $|k(i t)| \leq e^{-\sigma^{2} t^{2} / 4}$ for $|t| \leq \varepsilon_{0}$ with $\varepsilon_{0}>0$, hence $\left|k^{n}(i t / \sqrt{n})\right| \leq e^{-\sigma^{2} t^{2} / 4}$. If $|t / \sqrt{n}| \leq \varepsilon_{0}$ we have $C^{n} \leq \exp \left(t^{2} \varepsilon_{0}^{-2} \log C\right)$. Since $\left\|\left(Q^{i t / \sqrt{n}}\right)^{n}\right\| \leq\left|k^{n}(i t / \sqrt{n})\right|+\left\|R^{n}(i t / \sqrt{n})\right\|$ we have $\left\|\left(Q^{i t / \sqrt{n}}\right)^{n}\right\| \leq 2 e^{-A t^{2}}$ with $A=\sup \left(\sigma^{2} / 4, \varepsilon_{0}^{-2} \log C\right)$. 
Theorem 1.5 Assume that the Markov kernel $Q$ on $H^{\varepsilon}(X)$ is irreducible, $Q^{\text {it }}$ satisfies condition (D-F) for any $t \in \mathbb{R}$, the function $f$ on $X \times S$ is aperiodic with $\gamma(f)=0$, and $\int f^{2}(x, g) d m(x) d \mu(g)<\infty$. Then for any $\varphi \in H^{\varepsilon}(X)$ and arbitrary interval $I \subset \mathbb{R}$ :

$$
\lim _{n \rightarrow \infty} \sigma \sqrt{2 \pi n} \mathbb{E}_{x}\left(\varphi\left(s_{n} . x\right) 1_{I}\left(S_{n}(x, .)\right)\right]=m(\varphi) \ell(I),
$$

where $\ell$ is Lebesgue measure on $\mathbb{R}$.

Proof : As in [6] we consider the following sequence of Radon measures $\ell_{x}^{n}$ on $X \times \mathbb{R}$ defined by $\ell_{x}^{n}(\varphi \otimes u)=\sigma \sqrt{2 \pi n} \mathbb{E}_{x}\left(\varphi\left(s_{n} . x\right) u\left(S_{n}(x,).\right)\right)$ and we need to show the weak convergence of $\ell_{x}^{n}$ to $m \otimes \ell$. Hence it suffices to show that $\lim _{n \rightarrow \infty} \ell_{x}^{n}(\varphi \otimes u)=m(\varphi) \ell(u)$ for any continuous $u \in \mathbb{L}^{1}(\mathbb{R})$ with compactly supported Fourier transform $\widehat{u}$ (see $[6]$ ). The Fourier inversion formula gives with $\varepsilon>0$ :

$$
\begin{aligned}
& \mathbb{E}_{x}\left(\varphi\left(s_{n} . x\right) u\left(S_{n}(x, .)\right)=(2 \pi)^{-1} \int_{-\infty}^{\infty}\left(Q^{i t}\right)^{n} \varphi(x) \widehat{u}(t) d t\right. \\
& \ell_{x}^{n}(\varphi \otimes u)=\sigma \sqrt{n}(2 \pi)^{1 / 2}\left(\int_{|t| \geq \varepsilon^{\prime}}\left(Q^{i t}\right)^{n} \varphi(x) \widehat{u}(t) d t+\int_{-\varepsilon^{\prime} \sqrt{n}}^{\varepsilon^{\prime} \sqrt{n}}\left(Q^{i t / \sqrt{n}}\right)^{n} \varphi(x) \widehat{u}(t / \sqrt{n}) d t\right)
\end{aligned}
$$

Since $f$ is aperiodic, Proposition 1.3 gives that $Q^{i t}$ has no unimodular eigenvalue if $t \neq 0$. Since $Q^{i t}$ satisfies condition (D-F) it follows that the spectral radius of $Q^{i t}$ satisfies $r\left(Q^{i t}\right)<$ 1. Hence the first term in the right hand side has limit zero. On the other hand, the above lemma shows that for $\varepsilon^{\prime}$ small $\left(Q^{i t / \sqrt{n}}\right)^{n} \varphi(x) 1_{\left[-\varepsilon^{\prime} \sqrt{n}, \varepsilon^{\prime} \sqrt{n}\right]}(t)$ satisfies dominated convergence with limit $e^{-t^{2} \sigma^{2} / 2}$. The convergence of the second term to $m(\varphi) \int_{-\infty}^{\infty} e^{-\sigma^{2} t^{2} / 2} \widehat{u}(0) d t=$ $\left(\sigma^{-1} \sqrt{2 \pi}\right)^{-1} m(\varphi) \ell(u)$ follows.

Remark 1.6 Condition $(D-F)$ for $Q^{i t}(t \neq 0)$ and aperiodicity of $f$ are satisfied if $Q^{\text {it }}$ has spectral radius less than one and $f$ is non degenerate.

Clearly the same proof in the $\mathbb{L}^{2}$ context with $\mathbb{L}_{0}^{2}(X)=\left\{\varphi \in \mathbb{L}^{2}(X) ; m(\varphi)=0\right\}$ gives

Theorem 1.7 Assume that the Markov kernel $Q$ is irreducible and its spectral radius $r\left(Q_{0}\right)$ on $\mathbb{L}_{0}^{2}(X)$ satisfies $r\left(Q_{0}\right)<1$. Assume the Borel function $f(x, g)$ on $X \times S$ satisfies $\gamma(f)=0, \sigma^{2}(f)>0$, and the operator $Q^{i t}$ on $\mathbb{L}^{2}(X)$ has spectral radius less than 1 for any $t \neq 0$. Then for $\varphi \in \mathbb{L}^{2}(X)$ and arbitrary interval $I \subset \mathbb{R}:$

$$
\lim _{n \rightarrow \infty} \sigma \sqrt{2 \pi n} \mathbb{E}\left(\varphi\left(s_{n} . x\right) 1_{I}\left(S_{n}(x, .)\right)=m(\varphi) \ell(I) .\right.
$$

For various examples where the conditions of the theorem are satisfied, see [10]. Clearly the hypothesis and the result are stable under perturbation of $Q$ in the operator norm and this property gives new examples.

We observe that if $r\left(Q_{0}\right)<1$, Proposition 3.4 below allows one to verify that the spectral radius of $Q^{i t}$ is less than 1 in various situations. Then the spectral arguments developed in [25] can also be used for the proofs of various limit theorems, under a Hilbert space spectral hypothesis on $Q$. The condition $r\left(Q_{0}\right)<1$ of the theorem is called "strong mixing" of $Q$ 
in [45]. For a study of the central limit theorem in a similar general framework, we refer to [45]. The classical "Doeblin's condition" is stronger than "strong mixing". More precisely "Doeblin's condition" is equivalent to the fact that the spectral radius of $Q_{0}$ in $\mathbb{L}_{0}^{1}(m)$ is less than 1.

\section{Law of large numbers and spectral gap properties for prod- ucts of random matrices.}

In this section we describe some properties of products of random matrices which are essential for the proofs of limit theorems in sections 3, 4 below. In section 3 we will show the local limit theorem and large deviations asymptotics in a certain range for products of random matrices. These results will play also an important role in section 4 , in the study of extreme values for affine random walks. We need to give a brief exposition of known results on products of random matrices. We begin by describing some notation.

Let $\widetilde{G}=G L(V)$ be the full linear group of the Euclidean vector space $V=\mathbb{R}^{d}(d \geq 2)$, $G=S L(V)$ the special linear group of $V, \mathbb{P}(V)$ (resp $\mathbb{S}(V)$ ) the projective space (resp unit sphere) of $V$. The scalar product on $V$ will be written $\langle x, y\rangle$ if $x, y \in V$. The corresponding norm of $x \in V($ resp $g \in \widetilde{G})$ will be written $|x|($ resp $|g|)$. For $g \in \widetilde{G}$ we write $\gamma(g)=\sup \left(|g|,\left|g^{-1}\right|\right)$. If $x \in V$ we will denote $x($ resp $\tilde{x})$ its projection on $\mathbb{P}(V)(\operatorname{resp} \mathbb{S}(V))$. Also for $x \in \mathbb{P}(V)$ we will write $\tilde{x} \in \mathbb{S}(V)$ for one of its corresponding representatives in $\mathbb{S}(V)$. The action of $g$ on $x \in \mathbb{P}(V)$ (resp $y \in \mathbb{S}(V)$ ) will be written $g . x$ (resp g.y). We will use on $\mathbb{S}(V)(\operatorname{resp} \mathbb{P}(V)$ the distance $\bar{\delta}(\tilde{x}, \tilde{y})=|\tilde{x}-\tilde{y}|(\operatorname{resp} \bar{\delta}(x, y)=\inf (\tilde{\delta}(\tilde{x}, \tilde{y}), \tilde{\delta}(-\tilde{x}, \tilde{y}))$. We will also consider the distance $\delta$ on $\mathbb{P}(V)$ given by $\delta(x, y)=|x \wedge y|=\sin (x, y)$ which is equivalent to $\bar{\delta}$. We note that the natural norm $|x \wedge y|$ on the wedge product $\Lambda^{2} V$ satisfies $|x|^{2}|y|^{2}=|x \wedge y|^{2}+|\langle x, y\rangle|^{2}$. For $s \in \mathbb{R}$ we write $\bar{s}=\inf (s, 1), \hat{s}=\sup (s, 1)$. For a subset $E$ of a group we write $\langle E\rangle$ (resp $[E]$ ) for the closed subgroup (resp semigroup) generated by $E$. If $g \in \widetilde{G}$ has a simple dominant eigenvalue $\lambda_{g}$ we will write $V=\mathbb{R} v_{g} \oplus V_{g}^{<}$where $v_{g}$ is a dominant eigenvector and $V_{g}^{<}$the corresponding supplementary hyperplane. In this case we will say that $g$ is proximal and we denote by $g^{+} \in \mathbb{P}(V)$ the attracting fixed point defined by $v_{g}$. For a semigroup $\Gamma \subset \widetilde{G}$ we will write $\Gamma^{\text {prox }}$ for the subset of proximal elements in $\Gamma$. The closure of $\left\{g^{+} ; g \in \Gamma^{\text {prox }}\right\}$ will play an important role below in the discussion of aperiodicity for the function $f(x, g)=\log |g \widetilde{x}|$ with $g \in \widetilde{G}, x \in \mathbb{P}(V)$ (see Corollary 2.12). It will called limit set of $\Gamma$. The dual space of $V$ will be denoted $V^{*}$ and the dual map of $g$ by $g^{*}$. We will say that a semigroup $\Gamma \subset \widetilde{G}$ satisfies condition (I-P) if

a) No finite union of proper subspaces is $\Gamma$-invariant (strong irreducibility of $\Gamma$ ).

b) $\Gamma$ contains a proximal element.

It follows from [18] that condition (I-P) for $\Gamma,\langle\Gamma\rangle$ and the Zariski closure $Z c(\Gamma)$ of $\Gamma$ in $\widetilde{G}$ are all equivalent. Since $Z c(\Gamma)$ is a Lie subgroup of $\widetilde{G}$ with a finite number of connected components, condition (I-P) is easily verified. If the field $\mathbb{R}$ is replaced by the local field 
$\mathbb{Q}_{p}$, the corresponding (I-P) condition is useful (see [29]).

If $\Gamma$ acts on a locally compact metric space $X$ and if $\lambda($ resp $\nu)$ is a positive measure on $\Gamma($ resp $X)$ we define the convolution $\lambda * \nu$ by $(\lambda * \nu)(\varphi)=\int \varphi(\gamma \cdot x) d \lambda(\gamma) d \nu(x)$ where $\varphi \in C(X)$ is compactly supported. We will denote by $M^{1}(E)$ the space of probabilities on a Polish space $E$. In particular if $\mu \in M^{1}(\Gamma)$ we will write $\mu^{n}$ for its convolution power of order $n$. Also we will write $\mathbb{P}=\mu^{\otimes \mathbb{N}}$ for the corresponding probability on $\Gamma^{\mathbb{N}}$. The product of the random matrices $g_{k} \in \widetilde{G}(1 \leq k \leq n)$ will be written $S_{n}=g_{n} \cdots g_{1}$. The spectral radius of a bounded linear operator $U$ of a Banach space into itself will be denoted $r(U)=\lim _{n \rightarrow \infty}\left|U^{n}\right|^{1 / n}$. We begin by considering products of unimodular random matrices ; we follow ([4] [16], [22], [30]) with a few changes. We start with the following (see [22], $[52])$.

Theorem 2.1 Let $V=\mathbb{R}^{d}(d \geq 2), G=S L(V), \mu \in M^{1}(G)$ be such that the subgroup $<\operatorname{supp}(\mu)>$ is non-compact and strongly irreducible, and denote by $P_{\mu}$ the convolution operator defined by $\mu$ on the $G$-space $V \backslash\{0\}$. Let $\mathbb{L}^{2}(V)$ be the Hilbert space on $V$ with respect to Lebesgue measure and $r_{V}(\mu)$ the spectral radius of $P_{\mu}$ acting on $\mathbb{L}^{2}(V)$. Then $r_{V}(\mu)<1$.

We have the following corollaries.

Corollary 2.2 With $\mu$ as in the theorem and $\int \log |g| d \mu(g)<\infty$, the sequence $\frac{1}{n} \int \log |g| d \mu^{n}(g)$ converges to a positive number:

$$
\lim _{n \rightarrow \infty} \frac{1}{n} \int \log |g| d \mu^{n}(g) \geq-\frac{1}{d} \log r_{V}(\mu)>0 .
$$

Corollary 2.3 Assume $\operatorname{supp}(\mu)$ satisfies condition $(I-P)$, denote by $\bar{P}_{\mu}$ the operator on $\mathbb{P}(V)$ defined by convolution with $\mu$. Then $\bar{P}_{\mu}$ has a unique invariant probability measure $\nu$. If $\int \log |g| d \mu(g)$ is finite the sequence of functions on $\mathbb{P}(V), \frac{1}{n} \int \log |g x| d \mu^{n}(g)$ converges uniformly to $L_{\mu}=\int \log |g x| d \mu(g) d \nu(x)>0$.

This is a weak form of the well known Furstenberg's result giving exponential growth for products of unimodular random matrices : $\lim _{n \rightarrow \infty} \frac{1}{n} \log \left|g_{n} \cdots g_{1}\right|=L_{\mu}>0 \quad \mathbb{P}$ - a.e (see [16]). This fact follows also from the above and the subadditive ergodic theorem.

Corollary 2.4 We consider the d-dimensional torus $\mathbb{T}^{d}=\mathbb{R}^{d} / \mathbb{Z}^{d}(d>1)$, the action of a semigroup $\Gamma \subset S L(d, \mathbb{Z})$ on $\mathbb{T}^{d}$ by automorphisms, a probability measure $\mu \in M^{1}(\Gamma)$ such that the action of the group $<\operatorname{supp}(\mu)>$ on $\mathbb{R}^{d}$ is strongly irreducible. Then the spectral radius $r_{0}(\mu)$ of the corresponding convolution action on $\mathbb{L}_{0}^{2}\left(\mathbb{T}^{d}, m\right)$ where $m$ is Lebesgue measure, satisfies $r_{0}(\mu)<1$. 
We observe that this corollary (see[15]) is a very special case of a general result of automorphismactions on a compact nilmanifold $N / \Delta$ used below : the action of $\Gamma \subset \operatorname{Aut}(N / \Delta)$ on $\mathbb{L}_{0}^{2}(N / \Delta)$ does not contain weakly the identity representation if and only if there is no $\Gamma$-equivariant torus factor $T$ of $N / \Delta$ such that the corresponding quotient group acting on $T$ is virtually abelian (see [1]).

Corollary 2.5 With the hypothesis of Corollary 2.3 we assume that for some $\tau>0$, $\int|g|^{\tau} d \mu(g)$ is finite. Then there exists $\varepsilon_{0}>0$ such that for any $\left.\left.\varepsilon \in\right] 0, \varepsilon_{0}\right]$ :

$$
\lim _{n \rightarrow \infty} \sup _{|x|=1}\left(\int|g x|^{-\varepsilon} d \mu^{n}(g)\right)^{1 / n}<1 .
$$

Then in particular if $\operatorname{dim} V=2$, and $\left.2 \varepsilon \in] 0, \varepsilon_{0}\right]$ one has :

$$
\left.\rho(\varepsilon)=\lim _{n \rightarrow \infty} \sup _{\bar{x}, \bar{y} \in \mathbb{P}(V)} \int \frac{\delta^{\varepsilon}(g \cdot \bar{x}, g \cdot \bar{y})}{\delta^{\varepsilon}(\bar{x}, \bar{y})} d \mu^{n}(g)\right)<1,
$$

and the spectral radius of $\bar{P}_{\mu}$ on $H_{0}^{\varepsilon}(\mathbb{P}(V))=\left\{\varphi \in H^{\varepsilon}(\mathbb{P}(V)) ; \nu(\varphi)=0\right\}$ is at most $\rho(\varepsilon)<1$.

Remark 2.6 a) As the proof below shows, we can replace in the statement of the theorem $V$ by $\mathbb{Z}^{d}$, i.e $S L(V)$ by $S L(d, \mathbb{Z})$; then $r_{V}(\mu)$ is replaced by the spectral radius of the convolution by $\mu$ on $\mathbb{Z}^{d} \backslash\{0\}$, less than 1 under the same condition.

b) Strong irreducibility is a necessary hypothesis for the validity of the theorem : if $\mu=$ $\frac{1}{2}\left(\delta_{a}+\delta_{b}\right)$ with $a=\left(\begin{array}{cc}\lambda & 0 \\ 0 & 1 / \lambda\end{array}\right), b=\left(\begin{array}{cc}0 & 1 \\ -1 & 0\end{array}\right), \lambda>1$ we get with standard notations on $\mathbb{P}^{1}\left(\mathbb{R}^{2}\right): \nu=\frac{1}{2}\left(\delta_{\bar{e}_{1}}+\delta_{\bar{e}_{2}}\right), L_{\mu}=\lim _{n \rightarrow \infty} \frac{1}{n} \int \log |g| d \mu^{n}(g)=0$.

c) If $d=2$, condition (I-P) for $[\operatorname{supp}(\mu)]$ is equivalent to strong irreducibility and noncompactness of $<\operatorname{supp}(\mu)>$.

For the proof of the theorem we need the lemma (see [16]) :

Lemma 2.7 Assume that a semigroup $\Gamma \subset S L(V)$ is strongly irreducible and non-compact. Then there is no $\Gamma$-invariant probability measure on $\mathbb{P}(V)$.

Proof of Lemma 2.7 Assume $\nu \in M^{1}(\mathbb{P}(V))$ satisfies $g . \nu=\nu$ for every $g \in \Gamma$. Since $\Gamma$ is unbounded there exists $g_{n} \in \Gamma$ with $\lim _{n \rightarrow \infty}\left|g_{n}\right|=\infty$. We denote $u_{n}=\left|g_{n}\right|^{-1} g_{n}$ and we observe that $\left|\operatorname{det} u_{n}\right|=\left|g_{n}\right|^{-d}$ converges to zero. Since $\left|u_{n}\right|=1$, we can extract a convergent subsequence in End $V$, again denoted $u_{n}$ and we have $\lim _{n \rightarrow \infty} u_{n}=u$, with $|u|=1$, det $u=0$. We write $W=\operatorname{Ker} u, W^{\prime}=\operatorname{Im} u$ and we denote by $\bar{W}$ and $\bar{W}^{\prime}$ the corresponding projective subspaces with $\bar{W}^{c}=\mathbb{P}(V) \backslash \bar{W}$. We decompose $\nu$ as $\nu=\nu_{1}+\nu_{2}$ where $\nu_{1}\left(\operatorname{resp} \nu_{2}\right)$ is the restriction of $\nu_{1}\left(\operatorname{resp} \nu_{2}\right)$ to $\bar{W}^{c}(\operatorname{resp} \bar{W})$ and we observe that $\nu=$ 
$\lim _{n \rightarrow \infty} g_{n} . \nu=\lim _{n \rightarrow \infty} g_{n} . \nu_{1}+u . \nu_{2}$ where $u . \nu_{2}$ is supported by $\bar{W}^{\prime}$. Since $\mathbb{P}(V)$ is compact we can assume, taking a subsequence, that $g_{n} . \nu_{1}$ converges to $\nu_{1}^{\prime}$ which is supported on the subspace $\bar{W}_{1}=\lim _{n \rightarrow \infty} g_{n} \cdot \bar{W}$. Since $g . \nu=\nu$ it follows that $\nu\left(\bar{W}_{1} U \bar{W}^{\prime}\right)=1$. We consider the set $\Phi$ of subsets $F$ of $\mathbb{P}(V)$ which are finite unions of proper subspaces with $\nu(F)=1$. Since any decreasing sequence in $\Phi$ is finite, $\Phi$ has a least element $F_{0}$. Since $g . \nu=\nu$, we have $g . F_{0}=F_{0}$ for any $g \in \Gamma$, hence $\Gamma$ is not strongly irreducible. This gives the required contradiction.

Proof of Theorem 2.1 Assume $r_{V}(\mu)=1$ and let $z=e^{i \theta},|z|=1$ be a spectral value of $P_{\mu}=P$. Then either $\lim _{n \rightarrow \infty}\left\|P \varphi_{n}-z \varphi_{n}\right\|_{2}=0$ for some sequence $\varphi_{n} \in \mathbb{L}^{2}(V)$ with $\left\|\varphi_{n}\right\|_{2}=1$ or $\operatorname{Im}(P-z I)$ is not dense in $\mathbb{L}^{2}(V)$. In the second case, duality gives $z P^{*} \varphi=\varphi$ for some $\varphi \in \mathbb{L}^{2}(V), \varphi \neq 0$. Since $S L(V)$ preserves Lebesgue measure on $V, P$ is a contraction of $\mathbb{L}^{2}(V)$, hence the fixed points of $\bar{z} P$ and $z P^{*}$ in $\mathbb{L}^{2}(V)$ are the same i.e $P \varphi=z \varphi$. Hence it suffices to consider the first case. Then the condition $\lim _{n \rightarrow \infty}\left\|P \varphi_{n}-z \varphi_{n}\right\|_{2}=0$ is equivalent to $\lim _{n \rightarrow \infty}<P \varphi_{n}, z \varphi_{n}>=1$. Since $\left|P \varphi_{n}\right| \leq P\left|\varphi_{n}\right|$ and $\left\langle P\left|\varphi_{n}\right|,\left|\varphi_{n}\right|\right\rangle \leq 1$ we get $\lim _{n \rightarrow \infty}\left\langle P\left|\varphi_{n}\right|,\left|\varphi_{n}\right|\right\rangle=1$, i.e $\lim _{n \rightarrow \infty} \int\left\langle\left|\varphi_{n}\right| \circ g,\left|\varphi_{n}\right|\right\rangle d \mu(g)=1$. Since $\left\langle\left|\varphi_{n}\right| \circ g,\left|\varphi_{n}\right|\right\rangle \leq 1$ it follows that, taking a subsequence, $\lim _{n \rightarrow \infty}\left\langle\left|\varphi_{n}\right| \circ g,\left|\varphi_{n}\right|\right\rangle=1 \mu-$ a.e, i.e $\lim _{n \rightarrow \infty}\left\|\left|\varphi_{n}\right| \circ g-\left|\varphi_{n}\right|\right\|_{2}=0 \mu-$ a.e. On the other hand we have

$$
\left\|\left|\varphi_{n}\right|^{2} \circ g-\left|\varphi_{n}\right|^{2}\right\|_{1} \leq\left\|\left|\varphi_{n}\right| \circ g-\left|\varphi_{n}\right|\right\|_{2}\left\|\left|\varphi_{n}\right| \circ g+\left|\varphi_{n}\right|\right\|_{2} \leq 2\left\|\left|\varphi_{n}\right| \circ g-\left|\varphi_{n}\right|\right\|_{2},
$$

hence $\lim _{n \rightarrow \infty}\left\|\left|\varphi_{n}\right|^{2} \circ g-\left|\varphi_{n}\right|^{2}\right\|_{1}=0 \mu-$ a.e. We consider the probability $\nu_{n}$ on $V \backslash\{0\}$ defined by $\nu_{n}=\left|\varphi_{n}\right|^{2} \ell$, where $\ell$ is Lebesgue measure on $V$, and its projection $\bar{\nu}_{n}$ on $\mathbb{P}(V)$. Then we have in variation norm $\lim _{n \rightarrow \infty}\left\|g \nu_{n}-\nu_{n}\right\|=0$, hence $\lim _{n \rightarrow \infty}\left\|g \cdot \bar{\nu}_{n}-\bar{\nu}_{n}\right\|=0 \mu-$ a.e, and also $\lim _{n \rightarrow \infty} g \cdot \bar{\nu}_{n}-\bar{\nu}_{n}=0$ weakly for any $g \in \operatorname{supp}(\mu)$. Taking a weak limit $\nu$ of a subsequence $\bar{\nu}_{n_{k}}$ we get $g . \nu=\nu$ for any $g \in \operatorname{supp}(\mu)$, hence $\nu$ is $<\operatorname{supp}(\mu)>$-invariant. From the lemma this is impossible because $\Gamma=<\operatorname{supp}(\mu)>$ is non-compact and strongly irreducible.

Proof of Corollary 2.2 We have $1=\operatorname{det} g \leq|g|^{d}$, hence $|g| \geq 1$ for $g \in G$, and $\infty>\int \log |g| d \mu^{n}(g)=u_{n} \geq 0$. Also by subadditivity of $\log |g|: u_{m+n} \leq u_{m}+u_{n}$. It follows $n^{-1} u_{n} \leq u_{1}<\infty, \lim _{n \rightarrow \infty} n^{-1} u_{n}=\inf _{n \geq 1} n^{-1} u_{n}=c \geq 0$.

For $\varepsilon>0$ we consider the $\mathbb{L}^{2}$ functions $f_{\varepsilon}$ and $1_{C}$ on $V$ given by :

$$
f_{\varepsilon}(v)=\inf \left(1,|v|^{-d-\varepsilon}\right), C=\{1 \leq|v| \leq 2\} .
$$

The theorem gives :

$$
\limsup _{n \rightarrow \infty}\left|<P_{\mu}^{n} f_{\varepsilon}, 1_{C}>\right|^{1 / n} \leq r_{V}(\mu)<1
$$


But : $\left\langle P_{\mu}^{n} f_{\varepsilon}, 1_{C}\right\rangle \geq \int_{C}|g v|^{-d-\varepsilon} d \mu^{n}(g) d v \geq 2^{-d-\varepsilon} \ell(C) \int|g|^{-d-\varepsilon} d \mu^{n}(g)$. It follows $\log \left\langle P_{\mu}^{n} f_{\varepsilon}, 1_{C}\right\rangle \geq$ $-(d+\varepsilon) u_{n}+\log \left(2^{-d-\varepsilon} \ell(C)\right), \quad(d+\varepsilon) \lim _{n \rightarrow \infty} n^{-1} u_{n} \geq-\log r_{V}(\mu)$.

Hence $c \geq-d^{-1} \log r_{V}(\mu)>0$

Proof of Corollary 2.3 The uniqueness of the $\bar{P}_{\mu}$-invariant measure $\nu \in M^{1}(\mathbb{P}(V))$ will be shown later (Theorem 2.11).

This uniqueness implies the uniform convergence of the sequence $\frac{1}{n} \sum_{0}^{n-1} \bar{P}_{\mu}^{k} \varphi(x)$ to $\nu(\varphi)$, for any $\varphi \in C(\mathbb{P}(V))$. We can write :

$\int \log |g x| d \mu^{n}(g)=\int \log \left|g_{n} g_{n-1} \cdots g_{1} x\right| d \mathbb{P}(\omega)=\sum_{1}^{n} \int \log \left|g_{k}\left(g_{k-1} \cdots g_{1} . x\right)\right| d \mathbb{P}(\omega)=$

$\sum_{1}^{n} \int \log |g(h . x)| d \mu(g) d \mu^{k-1}(h)=\sum_{1}^{n} \bar{P}_{\mu}^{k-1} \varphi(x)$ with $\varphi(x)=\int \log |g x| d \mu(g)$.

The condition $\int \log |g| d \mu(g)<\infty$ implies the continuity of $\varphi$ on $\mathbb{P}(V)$. From above we get the uniform convergence of $\frac{1}{n} \int \log |g x| d \mu^{n}(g)$ to $\int \varphi(x) d \nu(x)$. The positivity of the limit follows from 2.2 .

Proof of Corollary 2.4 We observe that $m$ is preserved by any $\gamma \in S L(d, \mathbb{Z})$ and we can write $\mathbb{L}^{2}\left(\mathbb{T}^{d}\right)=\mathbb{L}^{2}\left(\mathbb{T}^{d}, m\right)$ as $\mathbb{L}^{2}\left(\mathbb{T}^{d}\right)=\mathbb{C} 1 \oplus \mathbb{L}_{0}^{2}\left(\mathbb{T}^{d}\right)$. For any $\varphi \in \mathbb{L}^{2}\left(\mathbb{T}^{d}\right)$ we have $T_{\mu} \varphi(x)=\mu * \varphi(x)=\sum_{\gamma} \varphi\left(\gamma^{-1} x\right) \mu(\gamma)$. We observe that the Fourier transform of $\varphi$, i.e $\widehat{\varphi}(k)=<\varphi, e_{k}>$ where $e_{k}(x)=e^{2 i \pi<k, x>}$ and $k \in \mathbb{Z}^{d}$, gives an isometry $\varphi \rightarrow \widehat{\varphi}$ from $\mathbb{L}^{2}\left(\mathbb{T}^{d}\right)$ to $\ell^{2}\left(\mathbb{Z}^{d}\right)$, in which $\mathbb{L}_{0}^{2}\left(\mathbb{T}^{d}\right)$ corresponds to $\ell^{2}\left(\mathbb{Z}^{d} \backslash\{0\}\right)$. Also for $g \in S L(d, \mathbb{Z})$, we have $\widehat{\varphi \circ g}=\widehat{\varphi} \circ g^{*}$. Clearly the stated hypothesis on $\langle\operatorname{supp}(\mu)>$ carries over to $<\operatorname{supp}(\mu)>^{*}$. If $\left\langle\operatorname{supp}(\mu)>\right.$ is strongly irreducible on $\mathbb{R}^{d}$, then $\langle\operatorname{supp}(\mu)\rangle \subset S L(d, \mathbb{Z})$ is infinite, hence non-compact. Hence we can use Theorem 2.1 and the Remark 2.6 to deduce that the spectral radius $r_{0}(\mu)$ of $T_{\mu}$ on $\mathbb{L}_{0}^{2}\left(\mathbb{T}^{d}\right)$ satisfies $r_{0}(\mu)<1$.

Proof of Corollary 2.5 We denote $u_{n}(\varepsilon)=\sup _{|x|=1} \int|g x|^{-\varepsilon} d \mu^{n}(g)$. Since $|g x| \geq$ $\left|g^{-1}\right|^{-1} \geq|g|^{-(d-1)}$ the integral is finite for $\varepsilon(d-1) \leq \tau$. We have $u_{m+n}(\varepsilon) \leq u_{m}(\varepsilon) u_{n}(\varepsilon)$ because :

$\int|g x|^{-\varepsilon} d \mu^{m+n}(g)=\int|g(h . x)|^{-\varepsilon}|h x|^{-\varepsilon} d \mu^{m}(g) d \mu^{n}(h) \leq \sup _{|y|=1} \int|g y|^{-\varepsilon} d \mu^{m}(g) \int|h x|^{-\varepsilon} d \mu^{n}(h)$

$\leq u_{m}(\varepsilon) u_{n}(\varepsilon)$. Hence $\lim _{n \rightarrow \infty}\left|u_{n}(\varepsilon)\right|^{1 / n}=\inf _{n}\left(u_{n}(\varepsilon)\right)^{1 / n}$. It follows that it suffices to show $u_{n}(\varepsilon)<1$ for some $n \geq 1$.

We write $|g x|^{-\varepsilon}=\exp (-\varepsilon \log |g x|)$ and use the inequality $e^{u} \leq 1+u+u^{2} e^{|u|}$ which gives

$$
\begin{gathered}
|g x|^{-\varepsilon} \leq 1-\varepsilon \log |g x|+\left(\varepsilon^{2} \log ^{2}|g|\right)|g|^{(d-1) \varepsilon} \\
\int|g x|^{-\varepsilon} d \mu^{n}(g) \leq 1-\varepsilon \int \log |g x| d \mu^{n}(g)+\varepsilon^{2} \int|g|^{(d-1) \varepsilon} \log ^{2}|g| d \mu^{n}(g) .
\end{gathered}
$$


The last integral is bounded by $\left(\int(\log |g|)^{4} d \mu^{n}(g)\right)^{1 / 2}\left(\int|g|^{2 \varepsilon(d-1)} d \mu^{n}(g)\right)^{1 / 2}$, hence is finite for any $n$ if $2 \varepsilon(d-1) \leq \tau$. Using Corollary 2.3, we fix $n$ such that $\int \log |g x| d \mu^{n}(g) \geq c>0$, hence $\liminf _{\varepsilon \rightarrow 0_{+}} \frac{1-u_{n}(\varepsilon)}{\varepsilon} \geq c>0$, i.e $u_{n}(\varepsilon)<1$ for $\varepsilon \leq \varepsilon_{0}$ small.

We observe that $\delta(\bar{x}, \bar{y})=|x \wedge y|$ where $|x|=|y|=1$ is also a distance on $\mathbb{P}(V)$ which is equivalent to $\bar{\delta}$ since $\delta(\bar{x}, \bar{y})=|\sin (x, y)|$. Also $\delta(g . \bar{x}, g \cdot \bar{y})=|g x||g y| \delta(\bar{x}, \bar{y})$ since $g \in$ $S L(2, V)$. If follows :

$$
\begin{aligned}
& \int \frac{\delta^{\varepsilon}(g \cdot \bar{x})(g \cdot \bar{y})}{\delta^{\varepsilon}(\bar{x}, \bar{y}} d \mu^{n}(g)=\int \frac{1}{|g x|^{\varepsilon}|g y|^{\varepsilon}} d \mu^{n}(g) \leq\left(\int|g x|^{-2 \varepsilon} d \mu^{n}(g)\right)^{1 / 2}\left(\int|g y|^{-2 \varepsilon} d \mu^{n}(g)\right)^{1 / 2} \leq \\
& \sup _{|x|=1} \int|g x|^{-2 \varepsilon} d \mu^{n}(g) .
\end{aligned}
$$

The inequality $\rho(\varepsilon)<1$ follows if $2 \varepsilon \leq \varepsilon_{0}$.

For any $\varphi \in H^{\varepsilon}(\mathbb{P}(V))$ we have $: \varphi(x)-\nu(\varphi)=\int(\varphi(x)-\varphi(y)) d \nu(y)$. It follows that $|\varphi-\nu(\varphi)|_{\infty} \leq[\varphi]_{\varepsilon}$, hence $\left|\bar{P}_{\mu}^{n} \varphi\right|_{\infty} \leq\left[\bar{P}_{\mu}^{n} \varphi\right]_{\varepsilon}$ if $\varphi \in H_{0}^{\varepsilon}(\mathbb{P}(V)$, and the norms $\varphi \rightarrow\|\varphi\|, \varphi \rightarrow$ $[\varphi]_{\varepsilon}$ on $H_{0}^{\varepsilon}(\mathbb{P}(V))$ are equivalent.

Also $\left[\bar{P}_{\mu}^{n} \varphi\right]_{\varepsilon} \leq[\varphi]_{\varepsilon} \sup _{\bar{x}, \bar{y} \in \mathbb{P}(V)} \int \frac{\delta^{\varepsilon}(g \cdot \bar{x}, g \cdot \bar{y})}{\delta^{\varepsilon}(\bar{x}, \bar{y})} d \mu^{n}(g)$. Since $\left|\bar{P}_{\mu}^{n} \varphi\right|_{\infty} \leq\left[\bar{P}_{\mu}^{n} \varphi\right]_{\varepsilon}$ on $H_{0}^{\varepsilon}(\mathbb{P}(V))$ we get $\left\|\bar{P}_{\mu}^{n}\right\| \leq \sup _{\bar{x}, \bar{y} \in \mathbb{P}(V)} \int \frac{\delta^{\varepsilon}(g \cdot \bar{x}, g \cdot \bar{y})}{\delta^{\varepsilon}(\bar{x}, \bar{y})} d \mu^{n}(g)$ on $H_{0}^{\varepsilon}(\mathbb{P}(V))$. The last conclusion follows from the above inequality $\rho(\varepsilon)<1$.

Corollary 2.5 has a natural extension to any dimension $d \geq 2$. The proof is based on a basic inequality between the two first Lyapunov exponents associated with $\mu \in M^{1}(G L(V))$ (see [18], [24]). We consider the ergodic dynamical system $(\Omega, \theta, \pi)$ where $\Omega=\widetilde{G}^{\mathbb{N}}, \theta$ is the shift, $\pi$ is a $\theta$-invariant probability, $f$ a $G L(d, \mathbb{R})$-valued function on $\Omega$ which satisfies $\int\left(\log ^{+}|f(\omega)|+\log ^{+}\left|f^{-1}(\omega)\right|\right) d \pi(\omega)<\infty$, and we write $S_{n}(\omega)=f\left(\theta^{n-1} \omega\right) \cdots f(\omega)$ for $n \geq 0$. Then the top Lyapunov exponent $L_{f}^{1}$ is defined by

$$
L_{f}^{1}=\lim _{n \rightarrow \infty} \frac{1}{n} \int \log \left|S_{n}(\omega)\right| d \pi(\omega)
$$

Using the wedge products $\Lambda^{k} V$ we define the other Lyapunov exponents $L_{f}^{k}$ by :

$$
L_{f}^{1}+\cdots+L_{f}^{k}=\lim _{n \rightarrow \infty} \frac{1}{n} \int \log \left|\Lambda^{k} S_{n}(\omega)\right| d \pi(\omega)
$$

Here we consider the two first Lyapunov exponents $L_{\mu}^{1}, L_{\mu}^{2}$ for products of independant random matrices with law $\mu$ i.e $\pi=\mu^{\otimes \mathbb{N}}=\mathbb{P}$. We have the following result (see [24]).

Theorem 2.8 Let $\mu \in M^{1}(G L(d, \mathbb{R}))$ be such that $\log \gamma(g)$ is $\mu$-integrable and the semigroup $[\operatorname{supp}(\mu)]$ of $G L(d, \mathbb{R})$ satisfies condition $(I-P)$. Then $L_{\mu}^{1}>L_{\mu}^{2}$. 
Condition (I-P) is satisfied for exemple, if the projection of the group $<\operatorname{supp}(\mu)>$ on $S L(d, \mathbb{R})$ is Zariski-dense in $S L(d, \mathbb{R})$ (see $[18]$ ).

The geometrical meaning of the theorem is that for any $x, y \in V \backslash\{0\}$, the angle between the random vectors $S_{n}(\omega) x, S_{n}(\omega) y$ decreases $\mathbb{P}$ - a.e to zero at exponential speed $\exp \left(L_{\mu}^{2}-\right.$ $\left.L_{\mu}^{1}\right)<1$.

The proof of this fact is based on a martingale argument due to H. Furstenberg and given below (see Theorem 2.11) : if $\nu$ is a $\bar{P}_{\mu}$-invariant probability on $\mathbb{P}(V)$ then the sequence of random measures $g_{1} \cdots g_{n} . \nu$ on $\mathbb{P}(V)$ converges weakly to a Dirac measure. The uniqueness of $\nu$ as a $\bar{P}_{\mu}$-invariant measure follows (see below). A useful algebraic tool for passing from ergodic properties of $\bar{P}_{\mu}$ to Lyapunov exponents is the following cocycle $\sigma$ : if $\xi \in T^{1}$ is a unit tangent vector to $\mathbb{P}(V)$ defined by $x \in V$ and $x \wedge y \in \wedge^{2}(V), \sigma(g, \xi)=\frac{|g(x \wedge y)||x|^{2}}{|g x|^{2}|x \wedge y|}$. Then, using the above convergence, one shows that, under condition (I-P) (see [24]) :

$$
\lim _{n \rightarrow \infty} \frac{1}{n} \sup _{\xi \in T^{1}} \int \log \sigma(g, \xi) d \mu^{n}(g)<0 .
$$

The extension of Corollary 2.4 is as follows :

Theorem 2.9 Assume that $\mu \in M^{1}(\widetilde{G})$ satisfies $\int \gamma^{\tau}(g) d \mu(g)<\infty$ for some $\tau>0$ and the semigroup $[\operatorname{supp}(\mu)]$ satisfies $(I-P)$. Then for $\varepsilon$ sufficiently small one has :

$$
\rho(\varepsilon)=\lim _{n \rightarrow \infty}\left(\sup _{\bar{x}, \bar{y}} \int \frac{\delta^{\varepsilon}(g \cdot \bar{x}, g \cdot \bar{y})}{\delta^{\varepsilon}(\bar{x}, \bar{y})} d \mu^{n}(g)\right)^{1 / n}<1 .
$$

In particular the spectral radius of $\bar{P}_{\mu}$ acting on $H_{0}^{\varepsilon}(\mathbb{P}(V))=\left\{\varphi \in H^{\varepsilon}(\mathbb{P}(V)) ; \nu(\varphi)=0\right\}$ is at most $\rho(\varepsilon)<1$.

In order to deduce limit theorems for products of random matrices (see [42]), we are led to consider the "Fourier operators" $\bar{P}_{\mu}^{i t}$ on $H^{\varepsilon}(\mathbb{P}(V))$ defined by $\bar{P}_{\mu}^{i t} \varphi(\bar{x})=\int|g x|^{i t} \varphi(g . \bar{x}) d \mu(g)$.

Corollary 2.10 We denote for any $k \geq 0, \varepsilon>0$ :

$$
C_{k}(\varepsilon)=\int|g|^{\varepsilon} d \mu^{k}(g), \rho_{k}(\varepsilon)=\sup _{\bar{x}, \bar{y} \in \mathbb{P}(V)} \int \frac{\delta^{\varepsilon}(g \cdot \bar{x}, g \cdot \bar{y})}{\delta^{\varepsilon}(\bar{x}, \bar{y})} d \mu^{k}(g)
$$

Then, for any $t \in \mathbb{R}, \varphi \in H^{\varepsilon}(\mathbb{P}(V)), k \geq 0$ :

$\left[\left(\bar{P}_{\mu}^{i t}\right)^{k} \varphi\right]_{\varepsilon} \leq \rho_{k}(\varepsilon)[\varphi]_{\varepsilon}+\left|\varepsilon^{-1} t\right| C_{k}(\varepsilon)|\varphi|_{\infty}$.

In particular for $\varepsilon$ small and $k$ large the operator $\bar{P}_{\mu}^{\text {it }}$ satisfies the $(D-F)$ inequality, $\left[\left(\bar{P}_{\mu}^{i t}\right)^{k} \varphi\right]_{\varepsilon} \leq \rho^{\prime}(\varepsilon)[\varphi]_{\varepsilon}+\left|\varepsilon^{-1} t\right| C^{\prime}(\varepsilon)|\varphi|_{\infty}$ with $\rho^{\prime}(\varepsilon)<1, C^{\prime}(\varepsilon)<\infty$

Proof of Theorem 2.9 We have if $|x|=|y|=1$,

$$
\frac{\delta(g \cdot \bar{x}, g \cdot \bar{y})}{\delta(\bar{x}, \bar{y})}=\frac{|g x \wedge g y|}{|g x||g y||x \wedge y|}
$$

Hence, by Schwarz inequality

$$
\left(\int \frac{\delta^{\varepsilon}(g . \bar{x}, g . \bar{y})}{\delta^{\varepsilon}(\bar{x}, \bar{y})} d \mu^{n}(g)\right)^{2} \leq \int\left(\frac{|g x \wedge g y|}{|g x|^{2}|x \wedge y|}\right)^{\varepsilon} d \mu^{n}(g) \int\left(\frac{|g x \wedge g y|}{|g y|^{2}|x \wedge y|}\right)^{\varepsilon} d \mu^{n}(g) .
$$


Hence it suffices to show

$$
\lim _{n \rightarrow \infty} \sup _{\xi \in T^{1}}\left(\int \sigma^{\varepsilon}(g, \xi) d \mu^{n}\right)^{1 / n}<1 .
$$

The proof is the same as for Corollary 2.5 where $|g x|^{-\varepsilon}$ replaces $\sigma^{\varepsilon}(g, \xi)$, once we have $\lim _{n \rightarrow \infty} \frac{1}{n} \sup _{\xi \in T^{1}} \int \log \sigma(g, \xi) d \mu^{n}(g)<0$ which, as explained above, follows from $L_{\mu}^{1}>L_{\mu}^{2}$. The spectral gap assertion on $H_{0}^{\varepsilon}(\mathbb{P}(V))$ is proved as in Corollary 2.5.

Proof of Corollary 2.10 We use the following mean value inequality for $|x|=|y|=1, t \in \mathbb{R}, \varepsilon \in] 0,1]:\left.|| g x\right|^{i t}-\left.|g y|^{i t}|\leq| \varepsilon^{-1} t|| g\right|^{\varepsilon}|x-y|^{\varepsilon}$.

Hence, with $\bar{x}, \bar{y} \in \mathbb{P}(V),|x|=|y|=1$ :

$$
\begin{aligned}
& \left.|| g x\right|^{i t}-\left.|g y|^{i t}|\leq| \varepsilon^{-1} t|| g\right|^{\varepsilon} \bar{\delta}^{\varepsilon}(\bar{x}, \bar{y}) \\
& \left.|| g x\right|^{i t} \varphi(g \cdot \bar{x})-\left.\left.|g y|^{i t} \varphi(g \cdot \bar{y})|\leq| \varphi\right|_{\infty}|| g x\right|^{i t}-|g y|^{i t} \mid+[\varphi]_{\varepsilon} \bar{\delta}^{\varepsilon}(g \cdot \bar{x}, g \cdot \bar{y}) .
\end{aligned}
$$

The first inequality follows by integration will respect of $\mu^{k}$. Let $\varepsilon>0$ with $0<2 \varepsilon<\varepsilon_{0}$ as in Corollary 2.5. Since $\lim _{k \rightarrow \infty}\left(\sup _{\bar{x}, \bar{y} \in \mathbb{P}(V)} \int \frac{\delta^{\varepsilon}(g \cdot \bar{x}, g \cdot \bar{y})}{\delta^{\varepsilon}(\bar{x}, \bar{y})} d \mu^{k}(g)\right)^{1 / k}<1$ we can fix $k>0$ with $\rho^{\prime}(\varepsilon)=\rho_{k}(\varepsilon)<1$.

Taking $\varepsilon$ with $\varepsilon<\tau$, we have $C_{k}(\varepsilon<\infty)$. Hence (D-F) inequality follows.

Theorem 2.11 Assume $\mu \in M^{1}(\widetilde{G}), \nu \in M^{1}(\mathbb{P}(V))$ is $\bar{P}_{\mu}$-invariant and $[\operatorname{supp}(\mu)]$ satisfies condition (I-P). Then the sequence $\sigma_{n} . \nu=g_{1} g_{2} \cdots g_{n} . \nu$ converges $\mathbb{P}$ - a.e to a Dirac measure $\delta_{z}(\omega)$. In particulier $\nu$ is unique as a $\bar{P}_{\mu}$-invariant probability and gives zero mass to any proper subspace of $\mathbb{P}(V)$.

The following corollaries will play an essential role in the proofs of the local limit theorem for $\log \left|S_{n}(\omega) x\right|$.

Corollary 2.12 Let $\Delta$ be a closed subsemigroup of $\widetilde{G}$ which satisfies condition (I-P). Then the action of $\Delta$ on $\mathbb{P}(V)$ has a unique minimal subset $\Lambda(\Delta)$ and $\Lambda(\Delta)$ is not contained in a countable union of proper subspaces. Furthermore $\Lambda(\Delta)$ is the closure of the set of attractive points of $\Delta$.

The set $\Lambda(\Delta)$ is called the limit set of $\Delta$.

Corollary 2.13 With the notations and hypothesis of Corollary 2.12 and $d \geq 2$, let $A \subset \Delta$ with $[A]=\Delta$, assume that $\varphi \in C(\mathbb{P}(V))$ satisfies for some $t \in \mathbb{R}, \theta \in[0,2 \pi[$ and any $\gamma \in A$, $\bar{x} \in \Lambda(\Delta),|\gamma x|^{i t} \varphi(\gamma . \bar{x})=e^{i \theta} \varphi(\bar{x})$.

Then $t=0, e^{i \theta}=1, \varphi=$ const. 
This corollary (see Proposition 3 in [24]) is useful in the verification of the aperiodicity conditions in the proofs of the local limit theorems. In view of its essential role in limit theorems we give a detailed proof below.

Corollary 2.14 Assume $\Gamma \subset G L(V)$ is a subsemigroup which satisfies (I-P), $d \geq 2$ and define $S_{\Gamma}=\left\{\log \left|\lambda_{g}\right| ; g \in \Delta^{\text {prox }}\right\}$. Then $S_{\Gamma}$ generates a dense subgroup of the additive group $\mathbb{R}$.

This property plays an essential role in the study of renewal theorems for products of random matrices (see [31]), and also in some geometric situations (see [7]).

For the proof of Theorem 2.11 we use the following lemmas.

Lemma 2.15 Let $\varphi \in C(\mathbb{P}(V))$ and denote $f_{n}(\omega)=\sigma_{n} \cdot \nu, f_{n}^{r}(\omega, \eta)=\sigma_{n} \eta_{1} \cdots \eta_{r} . \nu$ for $(\omega, \eta) \in \Omega \times \Omega$. Then for $r$ fixed the sequence $f_{n}^{r}(\omega, \eta)-f_{n}(\omega)$ converges $\mathbb{P} \times \mathbb{P}-$ a.e to zero.

Lemma 2.16 For any proper subspace $W$ of $\mathbb{P}(V)$ one has $\nu(W)=0$.

Proof of Lemma 2.15 Since $g \rightarrow g . \nu(\varphi)$ is $\mu$-harmonic, $f_{n}(\omega)$ is a martingale and we have for any $r \geq 0$ :

$$
\mathbb{E}\left(f_{n+r}-f_{n}\right)^{2}=\mathbb{E}\left(f_{n+r}^{2}\right)-\mathbb{E}\left(f_{n}^{2}\right) .
$$

Hence for any $N>0, \sum_{1}^{N} \mathbb{E}\left(f_{n+r}^{2}-f_{n}^{2}\right) \leq 2 r|\varphi|_{\infty}^{2}$. It follows that the series $\sum_{1}^{\infty} \mathbb{E}\left(f_{n+r}^{2}-f_{n}^{2}\right)$ is convergent. Also :

$$
\mathbb{E}\left(f_{n+r}^{2}-f_{n}^{2}\right)=\int\left|f_{n}^{r}(\omega, \eta)-f_{n}(\omega)\right|^{2} d(\mathbb{P} \otimes \mathbb{P})(\omega, \eta) .
$$

Hence the series $\sum_{1}^{\infty}\left|f_{n}^{r}(\omega, \eta)-f_{n}(\omega)\right|^{2}$ is $\mathbb{P} \otimes \mathbb{P}-$ a.e convergent. In particular for $r$ fixed and $\mathbb{P}$ - a.e $\eta=\left(\eta_{1}, \cdots, \eta_{r}\right)$ :

$\lim _{n \rightarrow \infty}\left|f_{n}^{r}(\omega, \eta)-f_{n}(\omega)\right|=0$

Proof of Lemma 2.16 We consider the set $\Phi$ of proper projective subspaces $H$ of $\mathbb{P}(V)$ of minimal dimension $r$ such that $\nu(H)>0$. Clearly if $H, H^{\prime} \in \Phi$ with $H \neq H^{\prime}$, then $\nu\left(H \cap H^{\prime}\right)=0$. It follows that for any $\varepsilon>0$ the set $\Phi_{\varepsilon}=\{H \in \Phi ; \nu(H) \geq \varepsilon\}$ is finite. In particular, there exists $H_{0} \in \Phi$ with $\nu\left(H_{0}\right)=\sup \{\nu(H) ; h \in \Phi\}$ and the set $\Phi^{\prime}=\left\{H \in \Phi ; \nu(H)=\nu\left(H_{0}\right)\right\}$ is finite. Since $g^{-1} H_{0}$ and $H_{0}$ have the same dimension $r$ the equation $\nu\left(H_{0}\right)=\int(g . \nu)\left(H_{0}\right) d \mu(g)$ implies $\nu\left(g^{-1} H_{0}\right)=\nu\left(H_{0}\right)$ for $\mu$-almost every $g$. Hence $g^{-1} H_{0} \in \Phi^{\prime}, g^{-1} \Phi^{\prime} \subset \Phi^{\prime}$ for any $g \in \operatorname{supp}(\mu)$. Since $\Phi^{\prime}$ is finite this contradicts strong irreducibility of $[\operatorname{supp} \mu]$. Hence $\Phi=\phi$. 
Proof of Theorem 2.11 Using Lemma 2.15 we know that the sequence $f_{n}(\omega)$ is a bounded martingale. The martingale convergence theorem implies the $\mathbb{P}-$ a.e convergence of $f_{n}(\omega)$. Since $C(\mathbb{P}(V))$ is separable, we get the weak convergence of $\sigma_{n} . \nu$ to the random probability $\nu_{\omega}$. We denote by $\Omega^{\prime} \subset \Omega$ the set of $\omega$ such that $\sigma_{n} . \nu$ converges weakly and such that the convergence in Lemma 2.15 for any $\varphi \in C(\mathbb{P}(V))$ takes place with $\mathbb{P} \otimes \mathbb{P}$-probability 1. Then, $\mathbb{P}\left(\Omega^{\prime}\right)=1$ and for any $\omega \in \Omega^{\prime}$, and for $\mathbb{P}-$ a.e $\eta$ :

$$
\lim _{n \rightarrow \infty} \sigma_{n} \eta_{1} \cdots \eta_{r} . \nu=\lim _{n \rightarrow \infty} \sigma_{n} \cdot \nu .
$$

Let $n_{k}(\omega)$ be a subsequence such that $\left|\sigma_{n_{k}}\right|^{-1} \sigma_{n_{k}}$ converges to a linear map $\tau_{\omega}$ with kernel $H_{\omega}$, hence the quasi projective map $\tau_{\omega}$ is defined and continuous on $\mathbb{P}(V) \backslash H_{\omega}$. Then by Lemma 2.16, $\nu$ and $\eta_{1} \cdots \eta_{r} . \nu$ gives zero mass to any proper subspace, hence $\tau_{\omega}\left(\eta_{1} \cdots \eta_{r} . \nu\right)=\tau_{\omega} \cdot \nu=\nu_{\omega}$

This equality is valid for a dense subset of $(\operatorname{supp}(\mu))^{\otimes r}$, hence for any $\gamma \in(\operatorname{supp}(\mu))^{r}$ : $\tau_{\omega}(\gamma . \nu)=\tau_{\omega} . \nu$. Then for any $\gamma$ in $[\operatorname{supp}(\mu)]$ we have $\tau_{\omega} \cdot(\gamma \cdot \nu)=\nu_{\omega}=\tau_{\omega} \cdot \nu$. Let $\gamma_{1}$ be a proximal element of $[\operatorname{supp}(\mu)]$ with $\gamma_{1}^{+}=x$, and $\gamma_{0} \in[\operatorname{supp}(\mu)]$ with $\gamma_{0} \cdot x \notin H_{\omega}\left(\gamma_{0}\right.$ exists by strong irreducibility of $[\operatorname{supp}(\mu)]$, see [19]). Then taking $\gamma=\gamma_{0} \gamma_{1}^{n} \in[\operatorname{supp}(\mu)]$ we get $\lim _{n \rightarrow \infty} \gamma_{0} \gamma_{1}^{n} . \nu=\gamma_{0} . \delta_{x}$. The continuity of $x \rightarrow \tau_{\omega} . x$ outside $H_{\omega}$ gives finally : $\tau_{\omega} . \nu=$ $\nu_{\omega}=\tau_{\omega}\left(\gamma_{0} . \delta_{x}\right)=\delta_{\tau_{\omega} \gamma_{0} . x}$. It follows that for $\omega \in \Omega^{\prime}, \nu_{\omega}$ is a Dirac measure $\delta_{z(\omega)}$ and $\tau_{\omega}\left(\mathbb{P}(V) \backslash H_{\omega}\right)=z(\omega)$. The martingale convergence theorem implies that the $\mathbb{P}$-law of $z(\omega)$ is $\nu$. Since $\tau_{\omega} . \nu=\delta_{z(\omega)}$ and $\tau_{\omega}$ is independant of $\nu$, this gives the uniqueness of $\nu$.

Proof of Corollary 2.12 Let $\mu \in M^{1}(\widetilde{G})$, be such that $[\operatorname{supp}(\mu)]=\Delta$, and $\nu \in$ $M^{1}(\mathbb{P}(V))$ be the unique $\bar{P}_{\mu}$-invariant probability. If $F$ is a closed $\Delta$-invariant subset of $\mathbb{P}(V)$, then Kakutani's fixed point theorem implies that there exists a $\bar{P}_{\mu}$-invariant probability $\eta \in M^{1}(F)$; the theorem gives $\eta=\nu$, hence $F \supset \operatorname{supp}(\eta)=\operatorname{supp}(\nu)$. The first assertion follows. If $\operatorname{supp}(\nu)$ is contained in a countable union of subspaces, then at least one of them has positive measure, which contradicts Theorem 2.11. The last assertion follows from the minimality of $\Lambda(\Delta)$.

Proof of Corollary 2.13 Let $\tilde{\Lambda}(\Delta)$ be the inverse image of $\Lambda(\Delta)$ in $V \backslash\{0\}$ and let $\tilde{\varphi}$ be the function on $\tilde{\Lambda}(\Delta)$ defined by $\tilde{\varphi}(v)=\varphi(\bar{v})|v|^{i t}$ where we can assume $t \geq 0$. Then the relation satisfied by $\varphi$ can be written as $\tilde{\varphi}(\gamma v)=e^{i \theta} \tilde{\varphi}(v)$, hence $\tilde{\varphi}(\delta v)=e^{i r \theta} \tilde{\varphi}(v)$ if $\delta \in A^{r}, r \in \mathbb{N}$. Assume $t>0$, hence $\rho=e^{2 \pi t}>1$. We have also $\tilde{\varphi}(\lambda v)=|\lambda|^{i t} \tilde{\varphi}(v)$ and in particular $\tilde{\varphi}\left( \pm \rho^{k} v\right)=\tilde{\varphi}(v), k \in \mathbb{Z}$. We observe that, since $\Delta$ acts minimally on $\Lambda(\Delta)$ and $\varphi$ is continuous, the invariance condition on $\varphi$ implies $|\varphi|=$ const, hence we can assume $|\varphi|=1$. It follows that the condition $\tilde{\varphi}(\lambda v)=\tilde{\varphi}(v)$ for some $v \in \tilde{\Lambda}(\Delta)$ is equivalent to $\lambda= \pm \rho^{k}(k \in \mathbb{Z})$. We define $\Lambda_{c} \subset \tilde{\Lambda}(\Delta)$ by $\Lambda_{c}=\tilde{\varphi}^{-1}(c)$ where $c$ is a value of $\tilde{\varphi}$ hence $\Lambda_{c}= \pm \rho^{\mathbb{Z}} \Lambda_{c} \neq \phi$. It follows that $\Lambda_{c}$ is closed as well as its projection in $\Lambda(\Delta)$. We note that for $\beta \in \mathbb{R}$, one has $\Lambda_{c e^{i \beta}}=\exp \left(\beta t^{-1}\right) \Lambda_{c}, \gamma\left(\Lambda_{c}\right) \subset \Lambda_{c e^{i \theta}}$ for $\gamma \in A$, hence $\delta\left(\Lambda_{c}\right) \subset \exp \left(r \theta t^{-1}\right) \Lambda_{c}$ if $\delta \in A^{r}$. Then the above projection of $\Lambda_{c}$ in $\Lambda(\Delta)$ is $\Delta$ invariant, hence equal to $\Lambda(\Delta)$ by minimality. Let $\gamma_{k}$ be sequence in $\Delta$ and $n_{k} \in \mathbb{Z}$ 
such that $u=\lim _{k \rightarrow \infty} \rho^{-n_{k}} \gamma_{k} \neq 0$. Then, since $\gamma_{k}\left(\Lambda_{c}\right) \subset \Lambda_{c e^{i \theta_{k}}}$ and $\pm \rho^{\mathbb{Z}} \Lambda_{c}=\Lambda_{c}$, we have also $u\left(\Lambda_{c}\right) \subset \Lambda_{c e^{i \alpha}} U\{0\}$ with $e^{i \alpha}=\lim _{k \rightarrow \infty} e^{i \theta_{k}}$, passing if necessary to a subsequence. Let $\delta \in A^{r}$ be a proximal element and $\gamma_{k}=\delta^{k}$, hence Keru $=W \neq\{0\}$ has codimension 1 and $\operatorname{Imu}=\mathbb{R} v_{\delta}$ for some $v_{\delta} \neq 0$. From the above we get $u\left(\Lambda_{c}\right) \subset \pm \rho^{\mathbb{Z}} v_{\delta} U\{0\}$. Since $u^{-1}\left(\left\{v_{\delta}\right\}\right)=a+W$ with $a \neq 0$, we get $\Lambda_{c} \backslash W \subset \pm \rho^{\mathbb{Z}}(a+W)$, hence $\Lambda_{c}$ has a countable projection in $V / W$. Let $\mathcal{F}_{c}$ be the family of subspaces $W^{\prime}$ in $V$ such that $\Lambda_{c}$ has a countable projection in $V / W^{\prime}$. We observe that the condition $\Lambda_{c e^{i \beta}}=\exp \left(\beta t^{-1}\right) \Lambda_{c}$ gives $\mathcal{F}_{c e^{i \beta}}=\mathcal{F}_{c}$ for $\beta \in \mathbb{R}$. Also if $W_{1}, W_{2} \in \mathcal{F}_{c}$, then $W_{1} \cap W_{2} \in \mathcal{F}$; this follows since $V / W_{1} \cap W_{2}$ is a subspace of $V / W_{1} \times V / W_{2}$. Then $\mathcal{F}_{c}$ has a minimum element $W_{c}=\underset{W^{\prime} \in \mathcal{F}_{c}}{\cap} W^{\prime}$. Since $\mathcal{F}_{c}=\mathcal{F}_{c e^{i \theta}}$, we have $W_{c}=W_{c e^{i \theta}}$, hence the condition $\gamma \Lambda_{c} \subset \Lambda_{c e^{i \theta}}$ for $\gamma \in A$ implies $\gamma\left(W_{c}\right) \subset W_{c}$. Since the projection of $\Lambda_{c}$ in $\mathbb{P}(V)$ is $\Lambda(\Delta)$ and, since $\operatorname{dim} V \geq 2$, we know from the theorem that $\Lambda(\Delta)$ is uncountable, it follows that $W_{c} \neq\{0\}$. This contradicts irreducibility of $\Delta$. Hence we have $t=0, \varphi(x)=e^{i \theta} \varphi(\gamma . x)$ for any $x \in \Lambda(\Delta), \gamma \in A$. Taking $\gamma$ proximal and $x=\gamma^{+}$we get $e^{i \theta}=1$ since $|\varphi(x)|=1$, hence $\varphi=$ const by minimality of the $\Delta$-action on $\Lambda(\Delta)$.

We need the following lemmas, the first of them is well known (see [5] p 90-94, Lemma 2.16). The second one is basic.

Lemma 2.17 Let $A$ be a finite set, $\Omega$ the compact metric space $A^{\mathbb{N}}$ and $\theta$ the shift on $\Omega$. For a function $\varphi$ on $\Omega$ we denote $S_{n}(\omega)=\sum_{k=0}^{n-1} \varphi \circ \theta^{k}(\omega)$. Suppose $\varphi$ is Hölder continuous and for any periodic point $\omega$ of period $p, S_{p} \varphi(\omega) \in \mathbb{Z}$. Then there exists a Hölder $\mathbb{Z}$-valued function $\varphi^{\prime}$ on $\Omega$ and a Hölder function $\psi$ on $\Omega$ such that $\varphi=\varphi^{\prime}+\psi-\psi \circ \theta$. If for any p-periodic $\omega$ we have $S_{p} \varphi(\omega)=0$, then $\varphi$ is of the form $\psi-\psi \circ \theta$ with $\psi$ Hölder on $\Omega$.

Lemma 2.18 Assume $g, h \in G L(V)$ are such that $h$ is proximal and $g . h^{+} \notin V_{h}^{<}$. Then for $n=2 p$ large, $g h^{n}$ is proximal and:

$$
\lim _{n \rightarrow \infty}\left(g h^{n}\right)^{+}=g \cdot h^{+}, \quad \lim _{n \rightarrow \infty} V_{g}^{<} h^{n}=V_{h}^{<} .
$$

Proof: We consider the sequence $u_{n}=\left|h_{n}\right|^{-1} h_{n}$ and we observe that $u_{n}$ converges to a map $\pi_{h}$ proportional to the projection on $\mathbb{R} v_{h}$ along the hyperplane $V_{h}^{<}$. Hence $\lim _{n \rightarrow \infty} g u_{n}=g \pi_{h}$ and $\operatorname{Im}\left(g \pi_{h}\right)=\mathbb{R}\left(g \cdot h^{+}\right), \operatorname{Ker}\left(g \pi_{h}\right)=V_{h}^{<}$. Since $g . h^{+} \notin V_{h}^{<}, g \pi_{h}$ is collinear to a projection onto a one-dimensional subspace. Since $g \pi_{h}$ has a simple dominant eigenvalue, the same is true for $g u_{n}$ for $n$ large. Hence for $n$ large, $g h^{n}$ is proximal and we have the required convergences.

Lemma 2.19 Assume $\Gamma \subset G L(V)$ is a subsemigroup which satisfies condition (I-P). Then there exists $a, b \in \Gamma^{\text {prox }}$ with $a^{+} \neq b^{+}, V_{a}^{<} \neq V_{b}^{<}$and $a^{+} \notin V_{b}^{<}, b^{+} \notin V_{a}^{<}$. 
Proof :We consider the dual semigroup $\Gamma^{*}$ acting on the dual space $V^{*}$ and the projective space $\mathbb{P}\left(V^{*}\right)$. Then condition (I-P) is also valid for $\Gamma^{*}$ and we can consider the corresponding limit set $\Lambda\left(\Gamma^{*}\right)$. We fix $a \in \Gamma^{\text {prox }}$ and observe that we can find $b \in \Gamma^{\text {prox }}$ with $V_{b}^{<} \neq V_{a}^{<}$, $a^{+} \notin V_{b}^{<}$. Otherwise there would be a dense subset of $\Lambda\left(\Gamma^{*}\right)$ contained in the union of the two projective subspaces in $\mathbb{P}\left(V^{*}\right)$ defined by $V_{a}^{<}$and $a^{+}$. Hence $\Lambda\left(\Gamma^{*}\right)$ would be contained in their union, but from Corollary 2.12, this is impossible. If $b^{+} \notin\left\{a^{+}\right\} \cup V_{a}^{<}$we have found the required pair $(a, b)$. If not, we consider $g \in \Gamma$ and the sequence $g b^{n}(n \in 2 \mathbb{N})$. By strong irreducibility we can choose $g \in \Gamma$ such that $g . b^{+} \notin V_{b}^{<} \cup V_{a}^{<} \cup\left\{a^{+}\right\}$. Then we apply the above lemma and replace $b$ by $g b^{n}=b^{\prime}$ for $n$ large. Then $V_{b^{\prime}}^{<}$is close to $V_{b}^{<}$and the above relations are still satisfied. Since $\left(b^{\prime}\right)^{+}$is close to $g . b^{+}$and $g . b^{+} \notin\left\{a^{+}\right\} \cup V_{a}^{<}$, the condition $\left(b^{\prime}\right)^{+} \notin\left\{a^{+}\right\} \cup V_{a}^{<}$is also satisfied. Hence we can take $\left(a, b^{\prime}\right)$ as the required pair.

Proof of Corollary 2.14 Since $\Gamma$ satisfies (I-P) we can choose $a_{1}, a_{2}$ in $\Gamma$ according to Lemma 2.19. Let $C_{1}, C_{2}$ be closed disjoint neighbourhoods of $a_{1}^{+}, a_{2}^{+}$in $\mathbb{P}(V)$ such that $\left(C_{1} \cup C_{2}\right) \cap\left(V_{a_{1}}^{<} \cup V_{a_{2}}^{<}\right)=\phi$ and let $0 \in \mathbb{P}(V)$ be a point outside $V_{a_{1}}^{<} \cup V_{a_{2}}^{<} \cup C_{1} \cup C_{2}$. Since $a, b$ are injective then for $i=1,2, \lim _{n \rightarrow \infty} a_{i}^{n} \cdot\left(C_{1} \cup C_{2}\right)=a_{i}^{+}, \lim _{n \rightarrow \infty} a_{i}^{n} .0=a_{i}^{+}$. Taking $n$ large and $a=a_{1}^{n}, b=a_{2}^{n}$ we get $a .0 \in C_{1}, b .0 \in C_{2}, a .\left(C_{1} \cup C_{2}\right) \subset$ Int $C_{1}, b .\left(C_{1} \cup C_{2}\right) \subset$ Int $C_{2}$. These relations imply that the semigroup $\Gamma(a, b)$ generated by $a, b$ is free, discrete, and any $\gamma \in \Gamma(a, b)$ is proximal. The limit set of $\Gamma(a, b)$ generates a subspace $W \subset V$ with dimension at least 2 and $\Gamma(a, b)$ satisfies condition (I-P) in $G L(W)$. In order to prove the corollary we can assume $\Gamma=\Gamma(a, b)$ and replace $V$ by $W$. Then we consider the $\{0,1\}$-valued metric $\delta$ on $\{a, b\}$ and endow $\Omega=\{a, b\}^{\mathbb{N}}$ with the metric $\widehat{\delta}\left(\omega, \omega^{\prime}\right)=\sum_{k=1}^{\infty} 2^{-k} \delta\left(\omega_{k}, \omega_{k}^{\prime}\right)$. We define a homeomorphism $z$ between $\Omega$ and $\Lambda(\Gamma)$ as follows. If $\omega=\left(a_{k}\right)_{k \in \mathbb{N}}$ then the sequence $a_{1} \cdots a_{n} .0$ converges to $z(\omega) \in C_{1} \cup C_{2}$ and we see that $z$ is a bi-Hölder homeomorphism ; hence we can transfer properties of $(\Omega, \theta)$ to the action of $\Gamma$ on $\Lambda(\Gamma)$. If $\tilde{z}(\omega) \in V$ with $|\tilde{z}(\omega)|=1$ has projection $z(\omega)$, then $\left|a_{1}(\omega) \tilde{z}(\theta \omega)\right|$ depends of $\omega$ only. It follows that, if we set $\varphi(\omega)=\log \left|a_{1}(\omega) \tilde{z}(\theta \omega)\right|, S_{n} \varphi(\omega)=\sum_{0}^{n-1} \varphi \circ \theta^{k}(\omega)$, we have, with $\gamma=a_{1} \cdots a_{n-1} \in \Gamma$ and $x=z\left(\theta^{n} \omega\right) \in \Lambda(\Gamma), S_{n} \varphi(\omega)=\log |\gamma x|$.

Then if $\psi$ is Hölder on $\Omega$, we define a Hölder function $\bar{\psi}$ on $\Lambda(\Gamma)$ by $\bar{\psi}(z(\omega))=\psi(\omega)$ and we have $\psi(\omega)-\psi\left(\theta^{n} \omega\right)=\bar{\psi}(\gamma \cdot x)-\bar{\psi}(x)$. In particular, if $\omega$ is periodic with period $p$, and $\varphi$ is as above then $\tilde{z}(\omega)$ is a dominant eigenvector of $\gamma=a_{1} \cdots a_{p-1}$ with $\log \left|\lambda_{\gamma}\right|=S_{p} \varphi(\omega)$. If $S_{\Gamma}=\{0\}$, then Lemma 2.17 implies the existence of $u$ continuous on $\Lambda(\Gamma)$ such that $|\gamma x| u(\gamma \cdot x)=u(x)$ for $\gamma \in \Gamma, x \in \Lambda(\Gamma)$, which contradicts Corollary 2.13.

If $S_{\Gamma}$ does not generate a dense subgroup of $\mathbb{R}$, then for some $c>0, S_{\Gamma} \subset c \mathbb{Z}$ hence $S_{p} \varphi(\omega) \in c \mathbb{Z}$ for any periodic point $\omega$ and we can apply Lemma 2.17 to the function $c^{-1} \varphi$. In particular the function $e^{2 i \pi c^{-1} \varphi}$ can be written in the form $e^{2 i \pi(\psi-\psi \circ \theta)}$ where $\psi$ is a Hölder function on $\Omega$. We can define $\bar{\psi}$ as above and write $u=e^{2 i \pi \bar{\psi}}$. Then $u$ is continuous and we have with $\gamma=a_{1} \cdots a_{n}, x=z\left(\theta^{n} \omega\right),|\gamma x|^{2 i \pi c^{-1}}=u(\gamma \cdot x) u^{-1}(x)$. Then we have for 
any $x \in \Lambda(\Gamma), \gamma \in \Gamma:|\gamma x|^{2 i \pi c^{-1}}=u(\gamma \cdot x) u^{-1}(x)$ hence Corollary 2.13 implies $2 i \pi c^{-1}=0$, and this is impossible.

Now we consider Lyapunov exponents and spectral gap properties for a more general situation than above (see [31]). They will be useful for large deviations results and for extreme value theory of affine random walks. Let $s \geq 0$ and let us define a kind a Mellin transform of $\mu \in M^{1}(G L(V))$, by $k(s)=\lim _{n \rightarrow \infty}\left(\int|g|^{s} d \mu^{n}(g)\right)^{1 / n}$. Since the function $g \rightarrow|g|^{s}$ is submultiplicative and $s \rightarrow|g|^{s}$ is Log-convex, $k(s)$ exists and is finite for $s$ in an interval $I_{\mu}$ of the form $\left[0, s_{\infty}\right]$ or $\left[0, s_{\infty}\left[\right.\right.$ with $k(s)=\infty$ for $s>s_{\infty}$. For $z \in \mathbb{C}$ the operator $P_{\mu}$ acting on $C(V \backslash\{0\})$ leaves invariant the space $C_{z}(V)$ of $z$-homogeneous continuous functions $f$ i.e $f \in C_{z}(V)$ means $f(\lambda v)=|\lambda|^{z} f(v)$ for $\lambda \neq 0, v \neq 0$. The action of $P_{\mu}$ on $C_{z}(V)$ reduces to an operator $P_{\mu}^{z}$ on $C(\mathbb{P}(V)):\left(P_{\mu}^{z} \varphi\right)(x)=\int|g \tilde{x}|^{z} \varphi(g . x) d \mu(g)$ where $\tilde{x} \in \mathbb{S}(V)$ has projection $x$ in $\mathbb{P}(V)$. We denote $z=s+i t$ where $s>0, t \in \mathbb{R}$ and our main result here is a spectral gap result for the operator $P_{\mu}^{s}$ (see [31]).

Theorem 2.20 Assume $d>1$ and the semigroup generated by $\operatorname{supp}(\mu)$ satisfies condition $(I-P)$. Then for any $s \in I_{\mu}$, there exists a unique probability measure $\nu^{s}$ on $\mathbb{P}(V)$, a unique positive continuous function $e^{s} \in C(\mathbb{P}(V))$ with $\nu^{s}\left(e^{s}\right)=1, P_{\mu}^{s} \nu^{s}=k(s) \nu^{s}, P_{\mu}^{s} e^{s}=k(s) e^{s}$. For $s \in I_{\mu}$, if $\int|g|^{s} \gamma^{\delta}(g) d \mu(g)<\infty$ for some $\delta>0$, then for $\varepsilon$ sufficiently small we have on $H^{\varepsilon}(\mathbb{P}(V)): P_{\mu}^{s}=k(s)\left(\nu^{s} \otimes e^{s}+U^{s}\right)$ where $\nu^{s} \otimes e^{s}$ is the projection on $\mathbb{C} e^{s}$ defined by $\varphi \rightarrow \nu^{s}(\varphi) e^{s}$ and $U^{s}$ is an operator with spectral radius less than 1 which satisfies $U^{s}\left(\nu^{s} \otimes e^{s}\right)=\left(\nu^{s} \otimes e^{s}\right) U^{s}=0$. Furthermore the function $k(s)$ is analytic, strictly convex on $] 0, s_{\infty}\left[\right.$ and the function $s \rightarrow \nu^{s} \otimes e^{s}$ from $] 0, s_{\infty}\left[\right.$ to $\operatorname{End}\left(H^{\varepsilon}(\mathbb{P}(V))\right.$ is analytic. If $t=\operatorname{Im} z \neq 0$, the spectral radius of $P_{\mu}^{z}$ is less than $k(s)$.

The proof follows the same lines as in Theorem 2.9. We give below corresponding indications.

The following lemmas and Propositions are basic.

Lemma 2.21 Assume $\sigma \in M^{1}(\mathbb{P}(V))$ is not supported by an hyperplane and $s>0$. There there exists $C_{s}(\sigma)>0$ such that for any $u \in$ End $V: \int|u x|^{s} d \sigma(x) \geq C_{s}(\sigma)|u|^{s}$

Proof: By homogeneity it suffices to show the above inequality if $|u|=1$.

The function $u \rightarrow \int|u x|^{s} d \sigma(x)$ is continuous on the unit sphere $\Sigma$ of End $V$. Since $\Sigma$ is compact, this function reaches its infimum $C_{s}(\sigma)$ at $u_{0} \in \Sigma$. If $C_{s}(\sigma)=0$, then $\int\left|u_{0} x\right| d \sigma(x)=0$, hence $\operatorname{supp}(\sigma) \subset$ Ker $u_{0}$, which contradicts the hypothesis on $\sigma$.

Lemma 2.22 If $s \in I_{\mu}$, there exists $\sigma \in M^{1}(\mathbb{P}(V)), k>0$ such that $P_{\mu}^{s} \sigma=k \sigma$. We have $k(s)=k$ and $\sigma$ is not supported on a hyperplane. Furthermore for any $n \in \mathbb{N}$ :

$$
\int|g|^{s} d \mu^{n}(g) \geq k^{n}(s) \geq C_{s}(\sigma) \int|g|^{s} d \mu^{n}(g)
$$


Proof: We consider the non linear operator $\widehat{P}^{s}$ on $M^{1}(\mathbb{P}(V))$ defined by $\widehat{P}^{s} \sigma=\left(P_{\mu}^{s} \sigma(1)\right)^{-1} P^{s} \sigma$. Since $s \in I_{\mu}$ this operator is continuous on the compact convex set $M^{1}(\mathbb{P}(V))$ endowed with the weak topology, hence Schauder-Tychonov theorem implies the existence of $k>0$ and $\sigma \in M^{1}(\mathbb{P}(V))$ with $\widehat{P}^{s} \sigma=\sigma, P_{\mu}^{s} \sigma=k \sigma$, hence $k=\left(P_{\mu}^{s} \sigma\right)(1)$.

For such a $\sigma$ the equation $k \sigma(\varphi)=\int \varphi(g . x)|g \tilde{x}|^{s} d \mu(g) d \sigma(x)$ implies $g . x \in \operatorname{supp}(\sigma), \mu-a . e$ if $x \in \operatorname{supp}(\sigma)$, hence for any $g \in \operatorname{supp}(\mu): g \cdot \operatorname{supp}(\sigma) \subset \operatorname{supp}(\sigma)$. Then the projective subspace $W$ generated by $\operatorname{supp}(\sigma)$ is $[\operatorname{supp}(\mu)]$-invariant and if $W$ is proper this contradicts condition (I-P). Since $k \leq\left(\int|g|^{s} d \mu^{n}(g)\right)^{1 / n}$ the two sided inequality for $k$ follows from Lemma 2.21, hence $k=k(s)$.

The dual version $\sigma^{\prime}$ of the measure $\sigma$ considered above is useful to construct a $P_{\mu^{-}}^{s}$ eigenfunction as follows.

Lemma 2.23 Assume $\sigma^{\prime} \in M^{1}\left(\mathbb{P}\left(V^{*}\right)\right)$ and $k^{\prime}>0$ satisfy ${ }^{*} P_{\mu}^{s} \sigma^{\prime}=k^{\prime} \sigma^{\prime}$. Then $k^{\prime}=k(s)$ and the function $e_{0}^{s}$ on $\mathbb{P}(V)$ defined by:

$$
e_{0}^{s}(x)=\int|\langle\tilde{x}, \tilde{y}\rangle|^{s} d \sigma^{\prime}(y)
$$

where $|\tilde{x}|=|\tilde{y}|=1$, satisfies $P_{\mu}^{s} e_{0}^{s}=k(s) e_{0}^{s}$. Furthermore $e_{0}^{s}$ is positive and Hölder of order $\bar{s}=\inf (s, 1)$

Proof : Since $\left|g^{*}\right|=|g|$, we have $\int|g|^{s} d \mu^{n}(g)=\int\left|g^{*}\right|{ }^{s} d \mu^{n}(g)$, hence using the dual version of Lemma 2.22 we get $k^{\prime}=k(s)$. Also :

$$
|g \tilde{x}|^{s} e_{0}^{s}(g \cdot x)=\int\left|\left\langle\tilde{x}, g^{*} \cdot \tilde{y}\right\rangle\right|^{s}\left|g^{*} \tilde{y}\right|^{s} d \sigma^{\prime}(y),{ }^{*} P_{\mu}^{s} \sigma^{\prime}=k^{\prime} \sigma^{\prime}
$$

hence, $P_{\mu}^{s} e_{0}^{s}(x)=k^{\prime} \int|\langle\tilde{x}, \tilde{y}\rangle|^{s} d \sigma^{\prime}(\tilde{y})=k(s) e_{0}^{s}(x)$. If $e_{0}^{s}(x)=0$ for some $x$, then $\langle\tilde{x}, \tilde{y}\rangle=0$ $\sigma^{\prime}$ - a.e, hence $\operatorname{supp}\left(\sigma^{\prime}\right)$ is contained in the hyperplane of $\mathbb{P}\left(V^{*}\right)$ orthogonal to $x$. Since $[\operatorname{supp}(\mu)]$ satisfies $(\mathrm{I}-\mathrm{P})$, this contradicts the dual version of Lemma 2.22. Hence $e_{0}^{s}$ is positive. The Hölder property of $e_{0}^{s}$ follows from the inequality $\left|a^{s}-b^{s}\right| \leq \widehat{s}|a-b|^{\bar{s}}$ where $a, b \in[0,1]$.

For $s \in I_{\mu}$ we define $q_{n}^{s}(x, g)=k^{-n}(s)|g \tilde{x}|^{s} e_{0}^{s}(g \cdot x)\left(e_{0}^{s}(x)\right)^{-1}, q^{s}(x, g)=q_{1}^{s}(x, g)$ and we observe that Lemma 2.23 implies $\int q_{n}^{s}(x, g) d \mu^{n}(g)=1$. Also we consider the Markov operator $Q^{s}$ on $\mathbb{P}(V)$ defined by $Q^{s} \varphi(x)=\int \varphi(g . x) q^{s}(x, g) d \mu(g)$ hence $\left(Q^{s}\right)^{n} \varphi(x)=$ $\int \varphi(g . x) q_{n}^{s}(x, g) d \mu^{n}(g)$. For $z=s+i t, t \in \mathbb{R}, s \in I_{\mu}$ we write :

$$
\left(Q^{z} \varphi\right)(x)=\int \varphi(g \cdot x) q^{s}(x, g)|g \tilde{x}|^{i t} d \mu(g) .
$$

Then we have the following simple lemmas which allow to control the iterates $\left(Q^{s}\right)^{n}$. 
Lemma 2.24 If $f \in C(\mathbb{P}(V))$ is real valued and satisfies $Q^{s} f \leq f$, then $f$ is constant on $\Lambda([\operatorname{supp} \mu])$ and equal to its infimum on $\mathbb{P}(V)$.

Lemma 2.25 For any $g \in S L(V) x, y \in V$ with $|x|=|y|=1$ :

$$
\begin{aligned}
& \left.|| g x\right|^{s}-\left.|g y|^{s}|\leq(s+1)| g\right|^{s} \tilde{\delta}^{\bar{s}}(x, y), \tilde{\delta}(g \cdot x, g \cdot y) \leq 2|g||g x|^{-1} \tilde{\delta}(x, y), \\
& \left|q^{s}(x, g)-q^{s}(y, g)\right| \leq b_{s}|g|^{s} k^{-1}(s) \tilde{\delta}^{\bar{s}}(x, y) \text { with } b_{s}>0 .
\end{aligned}
$$

To go further, in analogy with the case $s=0$ in Theorem 2.9, we need to consider a dynamical system $\left(\Omega, \theta, \mathbb{Q}^{s}\right)$ where $\Omega=\widehat{G}^{\mathbb{N}}, \theta$ is the shift and $\mathbb{Q}^{s}$ is a Markov measure defined as follows. We denote by $\pi^{s}$ a $Q^{s}$-invariant extremal probability on $\mathbb{P}(V)$, we observe that with $\omega=\left(g_{1}, \cdots, g_{n}\right) \in \Omega_{n}=\widetilde{G}^{\otimes n}, S_{n}(\omega)=g_{n} \cdots g_{1} \in \widetilde{G}$ we have $q_{n}^{s}\left(x, S_{n}(\omega)\right)=\prod_{k=1}^{n} q^{s}\left(s_{k-1} . x, g_{k}\right), \int q_{n}^{s}\left(x, S_{n}(\omega)\right) d \mu^{\otimes n}(\omega)=1$.

Definition 2.26 We denote by $\mathbb{Q}_{x}^{s} \in M^{1}(\Omega)$ the limit of the projective system of probabilities $q_{n}^{s}(x,.) \mu^{\otimes n}$ on $\Omega_{n}$, and we write $\mathbb{Q}^{s}=\int \mathbb{Q}_{x}^{s} d \pi^{s}(x)$.

We observe that $\mathbb{Q}^{s}$ is invariant under the shift $\theta$ on $\Omega$, we write $\mathbb{E}^{s}$ for the corresponding expectation : $\mathbb{E}^{s}(\varphi)=\int \mathbb{E}_{x}^{s}(\varphi) d \pi^{s}(x)$ where $\mathbb{E}_{x}^{s}$ is the expectation with respect to $\mathbb{Q}_{x}^{s}$. Since $\pi^{s}$ is extremal, it follows that $\mathbb{Q}^{s}$ is $\theta$-ergodic. Using the above Lemmas it is easy to show the following.

Proposition 2.27 There exists a constant $b>0$ such that for any $x, y \in \mathbb{P}(V)$ the total variation measure of $\mathbb{Q}_{x}^{s}-\mathbb{Q}_{y}^{s}$ is bounded by $b \delta^{\bar{s}}(x, y) \mathbb{Q}^{s}$. For any $x \in \mathbb{P}(V), \mathbb{Q}_{x}^{s}$ is equivalent to $\mathbb{Q}^{s}$.

Using the Markov operator ${ }^{*} Q^{s}$ on $\mathbb{P}\left(V^{*}\right)$, given by

$$
{ }^{*} Q^{s} \varphi(x)=\int{ }^{*} q^{s}(x, g) \varphi\left(g^{*} \cdot x\right) d \mu(g)
$$

where ${ }^{*} q^{s}$ is the dual analogue of $q^{s}$, the corresponding ${ }^{*} Q^{s}$-invariant measure ${ }^{*} \pi^{s}$ and function ${ }^{*} e_{0}^{s}$ on $\mathbb{P}\left(V^{*}\right)$, one obtains a ${ }^{*} P_{\mu}^{s}$-eigenmeasure by the formula ${ }^{*} \nu^{s}=\left({ }^{*} e_{0}^{s}\right)^{-1}{ }^{*} \pi^{s}$. Then the positive kernel $x \rightarrow \nu_{x}^{s}$ from $\mathbb{P}(V)$ to $\mathbb{P}\left(V^{*}\right)$ given by $\nu_{x}^{s}=\frac{|\langle x, .\rangle|^{s}}{e_{0}^{s}(x)} \nu^{s}$ is continuous in variation and satisfies $\int g^{*} \cdot \nu_{g \cdot x}^{s} q^{s}(x, g) d \mu(g)=\nu_{x}^{s}$, hence $S_{n}^{*} \cdot \nu_{S_{n} . x}^{s}$ is a bounded martingale with respect to $\mathbb{Q}_{x}^{s}$ and the natural filtration. Then, in analogy with Theorem 2.11 we have the following.

Proposition 2.28 The martingale $S_{n}^{*} . \nu_{S_{n} . x}^{s}$ converges $\mathbb{Q}_{x}^{s}$ - a.e to a Dirac measure $\delta_{z_{s}^{*}(\omega)}$, the law of $z_{s}^{*}(\omega)$ is ${ }^{*} \pi^{s},{ }^{*} \pi^{s}$ is proper, $g_{1}^{*} . z_{s}^{*}(\theta \omega)=z^{*}(\omega) \mathbb{Q}^{s}-$ a.e and if $<x, z^{*}(\omega)>\neq 0$, then $\lim _{n \rightarrow \infty} \sigma\left(S_{n}, \xi\right)=0 \mathbb{Q}^{s}$ - a.e. In particular, ${ }^{*} \pi^{s}$ is the unique ${ }^{*} Q^{s}$-invariant probability. 
Then we deduce the following (see [31]).

Theorem 2.29 The Lyapunov exponents $L_{\mu}^{1}(s), L_{\mu}^{2}(s)$ of $S_{n}(\omega)$ satisfy $L_{\mu}^{1}(s)>L_{\mu}^{2}(s)$.

One has, using the same arguments, the analogues of Theorem 2.9, Corollary 2.10. (See [31], Theorem 3.17).

Theorem 2.30 Assume that $\mu \in M^{1}(\tilde{G})$ satisfies $\int|g|^{s} \gamma^{\delta}(g) d \mu(g)<\infty$ for some $\delta>0$, $s \in I_{\mu}$ and $[$ supp $\mu]$ satisfies (I-P). Then for $\varepsilon$ sufficiently small one has

$$
\rho_{s}(\varepsilon)=\lim _{n \rightarrow \infty} \sup _{(x, y)}\left(\mathbb{E}_{x}^{s}\left(\frac{\delta^{\varepsilon}\left(S_{n} \cdot x, S_{n} \cdot y\right)}{\delta^{\varepsilon}(x, y)}\right)\right)^{1 / n}<1
$$

and the operator $Q^{s}$ on $H^{\varepsilon}(\mathbb{P}(V))$ satisfies $(D-F)$. For $z=s+i t, t \neq 0, s \in I_{\mu}$ the operator $Q^{z}$ on $H^{\varepsilon}(\mathbb{P}(V))$ satisfies $r\left(Q^{z}\right)<1$.

Then the spectral gap property of $P_{\mu}^{s}, P_{\mu}^{z}$ stated in Theorem 2.20 follows. The analyticity of the function $s \rightarrow k(s)$ follows from perturbation theory. The inequality $r\left(Q^{z}\right)<1$, hence $r\left(P_{\mu}^{z}\right)<k(s)$, is a consequence of the geometric Corollary 2.13.

\section{Local limit theorems for some transfer operators}

In this section we concentrate mainly on random walks on some homogeneous spaces. For analogous methods developed in the context of hyperbolic transformations or expanding transformations see [25], [7].

We apply the general local limit theorems $1.5,1.7$ to the specific situations detailed below.

For products of i.i.d matrices, with law $\mu \in M^{1}(\widetilde{G})$, and with the notations of section 1 we observe that precise large deviations can also be considered as local limit theorems for the function $f(x, g)=\log |g x|$ on $\widetilde{G} \times \mathbb{P}(V)$ and suitable centerings. We begin with the centering by $L_{\mu}^{1}$ which corresponds to $s=0$. In view of Theorem 2.9 and corollaries 2.10, 2.13, the conditions of Theorem 1.5 are satisfied, hence (see [42]) :

Theorem 3.1 Assume that $d>1$, the semigroup $[\operatorname{supp}(\mu)]$ satisfies condition $(I-P)$ and for some $\delta>0 \int \gamma^{\delta}(g) d \mu(g)<\infty$. Then there exists $\sigma>0$ such that for any $\varphi \in$ $C(\mathbb{P}(V)), x \in \mathbb{P}(V)$ and $\psi$ compactly supported on $\mathbb{R}$ and Riemann integrable, we have :

$$
\lim _{n \rightarrow \infty} \sigma \sqrt{2 \pi n} \int \varphi(g . x) \psi\left(\log |g x|-n L_{\mu}^{1}\right) d \mu^{n}(g)=\nu(\varphi) \ell(\psi) .
$$

Remark 3.2 In [19] the central limit theorem for the diagonal component of $S_{n}$ in the Cartan decompostion of $S L(V)$ is proved, under the condition of Zariski density of $[\operatorname{supp}(\mu)]$. In ([29], Theorem 3) this result is extended to a semi-simple group. The Gaussian law has full dimension and the proof is based on the fact that the set $\left\{\lambda_{\gamma} ; \gamma \in[\operatorname{supp}(\mu)]^{\text {prox }}\right\}$ is large 
a property which is stronger than the density property stated in corollary 2.14. This allows one to get a multidimensional local limit theorem which extends Theorem 3.1, under the condition that $[\operatorname{supp}(\mu)]$ has a Zariski dense projection in $S L(V)$.

Using the operators $Q^{s+i t}(s \geq 0, t \in \mathbb{R})$ and the probability $Q_{x}^{s}$ of section 2 instead of $\bar{P}_{\mu}$, $Q_{\mu}^{i t}$ and $\mathbb{P}$ we get in the same way, using Theorem 2.30 and the "anomalous" centering by $L_{\mu}^{1}(s)$ for $s>0$ :

Theorem 3.3 Assume $d>1$, $[\operatorname{supp}(\mu)]$ satisfies condition $(I-P)$ and for some $\delta>0, s \in$ $I_{\mu}, \int|g|^{s} \gamma^{\delta}(g) d \mu(g)$ is finite. Then there exists $\sigma^{s}>0$ such that for any $\varphi \in C(\mathbb{P}(V)), x \in$ $\mathbb{P}(V)$ and any compactly supported function $\psi$ on $\mathbb{R}$ which is Riemann integrable we have

$$
\lim _{n \rightarrow \infty} \sigma^{s} \sqrt{2 \pi n} k^{n}(s) \int|g x|^{s} \psi\left[\log |g x|-n L_{\mu}^{1}(s)\right] \quad \varphi(g \cdot x) d \mu^{n}(g)=\ell(\psi) e^{s}(x) \nu^{s}\left(\left(e^{s}\right)^{-1} \varphi\right) .
$$

This gives precise large deviations for the " anomalous mean values" $\gamma_{s}=L_{\mu}^{1}(s)$ of $\log \left|S_{n}(\omega) x\right|$. This corresponds to estimating the probability that $\log \left|S_{n}(\omega) x\right|$ belongs to an interval of fixed length centered at $n \gamma_{s}$, and gives exponential speed $k^{-n}(s)$ of convergence. One can show, using Theorem 2.9 and perturbation theory that there exists $s_{1}<0$ such that Theorem 3.2 remains walid for $s \in] s_{1}, s_{\infty}\left[\right.$. We recall that $L_{\mu}^{1}(s)=k^{\prime}(s) k^{-1}(s)$, hence the strict convexity of $\log k(s)$ implies that for $s \geq s_{1}, L_{\mu}^{1}(s)$ is increasing and takes only once any value between $L_{\mu}^{1}\left(s_{1}\right)$ and $\lim _{s \rightarrow s_{\infty}} L_{\mu}^{1}(s)$. However, in general for $s<0$, the function, $k(s)$ as defined above, loses its analyticity at a negative value of $s$, hence the analogue of Theorem 3.2 is no longer valid for any $s \in \mathbb{R}$, and a general $\mu$.

For an analogous statement see [43].

We consider now the following map $\theta$ of the circle $\mathbb{T}=\{|z|=1\} \subset \mathbb{C}$ defined by $\theta(z)=z^{2}$ and the $\mathbb{C}$-valued function $f(z)=z$. We are interested in the asymptotic properties of the Birkhoff sums $S_{n}(z)=\sum_{1}^{n} z^{2^{k}}=\sum_{0}^{n-1} f\left(\theta^{k} z\right)$.

Following [23], we sketch the proof of the following local limit in $\mathbb{C}$ :

Theorem 3.4 There exists a positive number $\sigma>0$ such that, for any bounded Borel set $B \subset \mathbb{C}$ with negligible boundary :

$$
\lim _{n \rightarrow \infty} 2 \pi n \sigma m\left\{z \in \mathbb{T} ; S_{n}(z) \in B\right\}=\ell(B)
$$

where $\ell(B)$ is the Lebesgue measure of $B$. In particular for $m-$ a.e $z \in \mathbb{T}$ the sums $S_{n}(z)$ are dense in $\mathbb{C}$.

Proof: We transfer the situation to the interval $I=[0,1]$, the map $\bar{\theta}(x)=\{2 x\}$ and the function $\bar{f}(x)=e^{2 i \pi x}=(\cos 2 \pi x, \sin 2 \pi x)$, replacing $m$ by Lebesgue measure $\bar{m}$ on $I$. Then the adjoint $Q$ of $\bar{\theta}$ with respect to $\bar{m}$ is given by $Q \varphi(x)=\frac{1}{2}(\varphi(a x)+\varphi(b x))$ where $a x=\frac{1}{2} x$, $b x=\frac{1}{2}(x+1)$. We write $x_{k}=a_{k} \cdots a_{1} x\left(a_{i}=a, b\right)$ for a trajectory of the Markov chain on $I$ defined by $Q$ and $\bar{S}_{n}(x)=\sum_{k=0}^{n-1} \bar{f}\left(x_{k}\right)$. By stationarity the laws of $S_{n}(z)$ and $\bar{S}_{n}(x)$ are the same, hence it suffices to show : 
$\lim _{n \rightarrow \infty} 2 \pi n \sigma \bar{m}\left\{x \in I ; \bar{S}_{n}(x) \in B\right\}=\ell(B)$.

As in [23] the recurrence and density properties of $S_{n}(z)$ follow.

The function $\bar{f}$ is $\mathbb{R}^{2}$-valued and we admit the multidimensional extension of Theorem 1.5. Here aperiodicity of $\bar{f}$ means that for $\lambda \in \mathbb{R}^{2} \backslash\{0\}, \alpha \in \mathbb{R}$ the equation :

(1) $e^{2 i \pi\langle\lambda, \bar{f}\rangle}=e^{2 i \pi \alpha}(u \circ a) u^{-1}=e^{2 i \pi \alpha}(u \circ b) u^{-1}$

has no Hölder solution $u$. We write here $Q^{i \lambda} \varphi=Q\left(e^{2 i \pi\langle\lambda, \bar{f}\rangle} \varphi\right)$. The Lipchitz coefficients of $\varphi, Q \varphi, Q^{i \lambda} \varphi$ satisfy.

$\left.[Q \varphi]_{1} \leq \frac{1}{2}[\varphi]_{1}, \mid Q^{i \lambda} \varphi\right]_{1} \leq \frac{1}{2}[\varphi]_{1}+2 \pi|\lambda||\varphi|_{\infty}$

hence condition (D-F) is satisfied by $Q, Q^{i \lambda}$ on $H^{1}(I), C(I)$. Clearly $Q$ is ergodic as is $\bar{\theta}$. Then, the local limit theorem will follow from Theorem 1.7 if $\bar{f}$ is aperiodic. Since $\bar{f}(0)=\bar{f}(1)$, from equation (1) we get $u(0)=u(1)$, hence we can write $u=e^{2 i \pi v}$ where $v$ is a Hölder 1-periodic function on $\mathbb{R}$. Then, using (1), the aperiodicity of $\bar{f}$ is not valid if there exists $\alpha \in \mathbb{R}$, such a $v$ and a $\mathbb{Z}$-valued function $k(x)$ with $\langle\lambda, \bar{f}\rangle(2 x)=$ $\alpha+v(x)-v(2 x))+k(x)$. By continuity we get that $k(x)$ is independant of $x$. Integrating with respect to $\bar{m}$, we get $\langle\lambda, \bar{f}\rangle(2 x)=v(x)-v(2 x)$. Now with $\lambda=(a, b) \neq(0,0)$ we write $\langle\lambda, \bar{f}\rangle(x)=a \cos 2 \pi x+b \sin 2 \pi x$, and we use the Fourier series expansion of $v$, $v(x)=\sum_{-\infty}^{\infty} c_{k} e^{4 i \pi k x}$. Then the above equation gives $a=c_{1}, b=c_{-1}, c_{0}=0, c_{2 k}=c_{k}$, $c_{2 k+1}=0$. Hence $c_{2^{k}}$ or $c_{-2^{k}}$ is equal to a or $b$, which contradicts the fact that $v$ is square integrable on $I$.

For the use of the Perron-Frobenius operator and spectral gaps on Sobolev spaces in the more general situation of holomorphic endomorphisms of the complex projective space $\mathbb{P}^{d}(\mathbb{C})$ see $[9]$ and $([11]$, Theorem 1.83).

The following is useful in dealing with $\mathbb{L}^{2}$-spectral gaps in the two situations which follow, i.e motion groups and nilmanifolds, but also in the general context of "strong mixing". For other examples see [10] where the following was proved in a slightly different form.

Proposition 3.5 Let $(X, m)$ be a probability space, $\Gamma$ a countable group acting on $X$ by measure preserving transformations, A a generating set for $\Gamma, \mu \in M^{1}(\Gamma)$ a probability measure on $\Gamma$ with $\operatorname{supp}(\mu)=A$. Let $c(a, x)=c_{a}(x)(a \in A, x \in X)$ be a measurable function with $|c(a, x)|=1, Q\left(\right.$ resp $\left.Q^{c}\right)$ the operator on $\mathbb{L}^{2}(X, m)=\mathbb{L}^{2}(X)$ defined by $Q \varphi=\sum_{a \in A} \mu(a) \varphi \circ a\left(\right.$ resp $\left.Q^{c} \varphi=\sum_{a \in A} \mu(a) c_{a} \varphi \circ a\right)$. Assume that the restriction $Q_{0}$ of $Q$ to $\mathbb{L}_{0}^{2}(X)$ satisfies $r\left(Q_{0}\right)<1$. Then one has $r\left(Q^{c}\right)=1$ if and only if there exists a measurable function $\alpha(x)$ with value in $[0,2 \pi[$ and $\theta \in[0,2 \pi[$ such that for any $a \in A$ $c(a, x)=e^{i(\alpha(a x)-\alpha(x)+\theta)} m$-a.e. In particular any unimodular spectral value of $Q^{c}$ is an eigenvalue of $Q^{c}$.

Proof : The proof begins like the proof of Theorem 2.1. Assume $r\left(Q^{c}\right)=1$ and let $e^{i \theta}$ be a spectral value of $Q^{c}$. Then, either the subspace $\operatorname{Im}\left(e^{i \theta}-Q^{c}\right)$ is not dense in $\mathbb{L}^{2}(X)$ or there exists $\varphi_{n} \in \mathbb{L}^{2}(X)$ with $\left\|\varphi_{n}\right\|_{2}=1$ such that $\lim _{n \rightarrow \infty}\left\|Q^{c} \varphi_{n}-e^{i \theta} \varphi_{n}\right\|_{2}=1$. In the first 
case there exists $\varphi \in \mathbb{L}^{2}(X)$ with $e^{i \theta}\left(Q^{c}\right)^{*} \varphi=\varphi$. Since $e^{-i \theta} Q^{c}$ is a contraction of $\mathbb{L}^{2}(X)$, $\varphi$ is also a fixed point of $e^{-i \theta} Q^{c}$, hence $Q^{c} \varphi=e^{i \theta} \varphi$ and it suffices to consider the second case . We have :

$$
\begin{aligned}
& 0 \leq\left\|Q^{c} \varphi_{n}-e^{i \theta} \varphi_{n}\right\|_{2}=\left\|Q^{c} \varphi_{n}\right\|_{2}^{2}+\left\|\varphi_{n}\right\|_{2}^{2}-2 \operatorname{Re}\left\langle Q^{c} \varphi_{n}, e^{i \theta} \varphi_{n}\right\rangle, \\
& 0 \leq 2-2 \operatorname{Re}\left\langle Q^{c} \varphi_{n}, e^{i \theta} \varphi_{n}\right\rangle .
\end{aligned}
$$

Then the condition $\lim _{n \rightarrow \infty}\left\|Q^{c} \varphi_{n}-e^{i \theta} \varphi_{n}\right\|=0$ is equivalent to $\lim _{n \rightarrow \infty} \operatorname{Re}\left\langle Q^{c} \varphi_{n}, e^{i \theta} \varphi_{n}\right\rangle=1$ hence to $\lim _{n \rightarrow \infty}\left\langle Q^{c} \varphi_{n}, e^{i \theta} \varphi_{n}\right\rangle=1$. Since $\left\|\varphi_{n}\right\|_{2}=1$ and $|c(a, x)|=1$ we have :

$|Q \varphi|(x) \leq Q|\varphi|(x), 0 \leq\left|\left\langle Q^{c} \varphi_{n}, e^{i \theta_{n}} \varphi_{n}\right\rangle\right| \leq<Q\left|\varphi_{n}\right|,\left|\varphi_{n}\right|>\leq 1$.

It follows $\lim _{n \rightarrow \infty}\left\langle Q\left|\varphi_{n}\right|,\left|\varphi_{n}\right|\right\rangle=1$, i.e $\lim _{n \rightarrow \infty}\left\|Q\left|\varphi_{n}\right|-\left|\varphi_{n}\right|\right\|_{2}=0$.

We can write $\left|\varphi_{n}\right|=\left\langle\left|\varphi_{n}\right|, 1\right\rangle+\psi_{n}$ where $\psi_{n}:=\left|\varphi_{n}\right|-\left\langle\left|\varphi_{n}\right|, 1\right\rangle 1 \in \mathbb{L}_{0}^{2}(X)$, hence $\lim _{n \rightarrow \infty} \|(Q-$ $I) \psi_{n} \|_{2}=0$ and $\left\langle\left|\varphi_{n}\right|, 1\right\rangle \leq\left\|\varphi_{n}\right\|_{2}=1,1=\left\langle\varphi_{n}, 1\right\rangle^{2}+\left\|\psi_{n}\right\|_{2}^{2}$.

Since $r\left(Q_{0}\right)<1$, the restriction of $Q-I$ to $\mathbb{L}_{0}^{2}(X)$ is invertible, hence the condition $\lim _{n \rightarrow \infty}\left\|(Q-I) \psi_{n}\right\|_{2}=0$ implies $\lim _{n \rightarrow \infty}\left\|\psi_{n}\right\|_{2}=0$. Hence $\lim _{n \rightarrow \infty}\left\|\left|\varphi_{n}\right|-1\right\|_{2}=0$. On the other hand, the condition $\lim _{n \rightarrow \infty}\left\langle Q^{c} \varphi_{n}, e^{i \theta} \varphi_{n}\right\rangle=1$ can be written as $\lim _{n \rightarrow \infty} \sum_{a \in A}\left\langle c_{a} \varphi_{n} \circ\right.$ $\left.a, e^{i \theta} \varphi_{n}\right\rangle \mu(a)=1$ with $\left|\left\langle c_{a} \varphi_{n} \circ a, e^{i \theta} \varphi_{n}\right\rangle\right| \leq 1$ for any $a \in A$. It follows $\lim _{n \rightarrow \infty} \| c_{a} \varphi_{n} \circ a-$ $e^{i \theta} \varphi_{n} \|_{2}=0$.

We write $\varphi_{n}=\left|\varphi_{n}\right| e^{i \alpha_{n}}$ with $\alpha_{n}(x) \in[0,2 \pi[$, hence :

$$
c_{a} \varphi_{n} \circ a-e^{i \theta} \varphi_{n}=c_{a} e^{i \alpha_{n} \circ a}\left(\left|\varphi_{n} \circ a\right|-1\right)-e^{i \theta}\left(\left|\varphi_{n}\right|-1\right) e^{i \alpha_{n}}+\left(c_{a} e^{i \alpha_{n} \circ a}-e^{i\left(\alpha_{n}+\theta\right)}\right) \text {. }
$$

From above we get $\lim _{n \rightarrow \infty}\left\|c_{a} e^{i\left(\alpha_{n} \circ a-\alpha_{n}-\theta\right)}-1\right\|_{2}=0$, therefore for a subsequence $n_{k}$ :

$$
c_{a}(x) e^{-i \theta}=\lim _{k \rightarrow \infty} e^{-i\left(\alpha_{n_{k}}(a x)-\alpha_{n_{k}}(x)\right.}, m-\text { a.e. }
$$

Hence for any $\gamma \in\langle A\rangle=\Gamma, \sigma(\gamma, x)=\lim _{k \rightarrow \infty} e^{-i\left(\alpha_{n_{k}}(\gamma x)-\alpha_{n_{k}}(x)\right)}$ exists $m-$ a.e and is a $\mathbb{T}$-valued cocycle. On the other hand, the spectral gap property $r\left(Q_{0}\right)<1$ implies the strong ergodicity (see [47] Proposition 2.10) of the action of $\Gamma$ on $X$, hence proposition 2.3 of [47] gives the existence of a measurable function $\alpha$ on $X$ such that :

$$
\sigma(\gamma, x)=c_{\gamma}(x) e^{-i|\gamma| \theta}=e^{-i(\alpha(\gamma x)-\alpha(x))}, m-\text { a.e }
$$

with $|\gamma|$ equal to the length of $\gamma$ in $A$. The result follows.

Remark 3.6 The proof of [47] shows that, up to unimodular coefficients, the sequence $\varphi_{n}$ converges in measure to an eigenfunction $\varphi$ of $Q^{c}$ with eigenvalue $e^{i \theta}$.

We write the group of complex motions of $\mathbb{C}^{d}$ in semi-direct product form $M_{d}=S U(d) \alpha \mathbb{C}^{d}$, we denote by $\mu$ a finitely supported probability on $M_{d}$, and by $\bar{\mu}$ its projection on $S U(d)$; let $B=\operatorname{supp}(\mu), A=\operatorname{supp}(\bar{\mu})$. If $A$ generates a dense subgroup of $S U(d)$, i.e $\langle A\rangle=S U(d)$, and if the elements of $A$ have algebraic coefficients in some base of $\mathbb{C}^{d}$, then it is known (see [3]) that for $d>1$ the convolution operator $\rho_{0}(\bar{\mu})$ by $\bar{\mu}$ on $\mathbb{L}_{0}^{2}\left(\mathbb{S}^{2 d-1}\right)$ satisfies $r\left(\rho_{0}(\bar{\mu})\right)<1$. For $d=1$, the problem of equidistribution of $\mu^{n} * \delta_{v}(v \in \mathbb{C})$ was considered by D.A Kazdhan ([36]). In this case $M_{2}$ is solvable and $\bar{\mu}$ defines a random walk on $\mathbb{T}$, which has no spectral 
gap in $\mathbb{L}^{2}(\mathbb{T})$, in general ; however $M_{2}$ is non-abelian and unitary representations are still useful (see [21], [53]) and allow one to obtain equidistribution. For $d>1$ the analogous problem remained unsolved for a long time, and the corresponding question was asked by G.-A Margulis. Using the result of [3] we give an affirmative answer (see [10]). We note that the same question has been recently solved in full generality in [51] i.e without using the spectral gap property in $S U(d)$. Our method here is of general interest in the larger context of Markov operators with a Hilbert space spectral condition, i.e $Q$ strongly mixing in the sense of [45].

Theorem 3.7 With the above notations and $d>1$ assume that $\langle B\rangle=M_{d}$ and $A \subset S U(d)$ consists of matrices with algebraic coefficients. Then there exists $\sigma(\mu)>0$ such that for any continuous compactly supported function $\varphi$ on $\mathbb{C}^{d}$, any $v \in \mathbb{C}^{d}$,

$\lim _{n \rightarrow \infty} \sigma(\mu)(2 \pi n)^{d}\left(\mu^{n} * \delta_{v}\right)(\varphi)=\ell(\varphi)$

where $\ell$ is Lebesgue measure on $\mathbb{C}^{d} \simeq \mathbb{R}^{2 d}$.

Proof : We recall some notations from unitary representations of $M_{d}$, where $\mathbb{C}^{d}$ is indentified with $V=\mathbb{R}^{2 d}$. The Fourier transform of $\varphi \in \mathbb{L}^{1}(V)$ is given by $\widehat{\varphi}(x)=$ $\int \varphi(v) e^{2 i \pi\langle x, v\rangle} d \ell(v)$. For $x=t u$ with $t>0, u \in \mathbb{S}^{2 d-1}$ we write $\varphi_{t}(u)=\widehat{\varphi}(t u)$ so that the Fourier inversion formula for $\varphi$ gives $\varphi(v)=\int \varphi_{t}(u) e^{-2 i \pi t\langle u, v\rangle} d \ell(x)$. If $h \in M_{d}$ is given by $h v=a v+b$ where $a \in S U(d), b \in V$, we get $\varphi\left(h^{-1} v\right)=\int \varphi_{t}\left(a^{-1} u\right) e^{2 i \pi t\langle u, b\rangle} e^{-2 i \pi t\langle u, v\rangle} d \ell(x)$ so that the function $\varphi \circ h^{-1}$ has $t$-Fourier component given by

$$
\left(\rho_{t}(h) \varphi_{t}\right)(u)=\varphi_{t}\left(a^{-1} u\right) e^{2 i \pi t\langle u, b\rangle}
$$

For $t>0$ the map $h \rightarrow \rho_{t}(h)$ is a unitary representation of $M_{d}$ in the Hilbert space $\mathbb{L}^{2}\left(\mathbb{S}^{2 d-1}\right)$ which is irreducible. We denote by $Q\left(\operatorname{resp} Q^{i t}\right)$ the operator on $\mathbb{L}^{2}\left(\mathbb{S}^{2 d-1}\right)$ given by

$$
Q \varphi(u)=\sum_{a \in A} \varphi\left(a^{-1} u\right) \bar{\mu}(a)\left(\operatorname{resp} Q^{i t} \varphi(u)=\sum_{h \in B} e^{2 i \pi t\langle u, b\rangle} \varphi\left(a^{-1} u\right) \mu(h)\right)
$$

so that $Q^{i t}=\rho_{t}(\mu), \check{\mu}^{n} * \delta_{v}(\varphi)=\int\left(\left(Q^{i t}\right)^{n} \varphi_{t}\right)(u) e^{-2 i \pi t\langle u, v\rangle} d \ell(x)$. With the notation of Proposition 3.4 we have $X=\mathbb{S}^{d-1}, Q^{i t}=Q^{c}$ where $c(u, h)=e^{2 i \pi t\langle u, b\rangle}$.

In view of Theorem 1.7, in order to prove the statement, it suffices to show that the restriction $Q_{0}$ of $Q$ to $\mathbb{L}_{0}^{2}\left(\mathbb{S}^{2 d-1}\right)$ satisfies $r\left(Q_{0}\right)<1$, and $r\left(Q^{i t}\right)<1$ if $t \neq 0$. Since $<A\rangle=S U(d)$ and $A$ has algebraic coefficients the first assertion follows from [3]. Using strict convexity in $\mathbb{L}^{2}\left(\mathbb{S}^{2 d-1}\right)$, the second assertion follows from Proposition 3.4 if there is no function $\varphi_{t} \in \mathbb{L}^{2}\left(\mathbb{S}^{2 d-1}\right)$ such that for some $\theta \in \mathbb{R}, t>0$, any $h \in B: \rho_{t}\left(h^{-1}\right) \varphi_{t}=e^{i \theta} \varphi_{t}$. But this relation implies that the group $\rho_{t}(\langle B\rangle)=\rho_{t}\left(M_{d}\right)$ has a one dimensional invariant subspace ; since the representation $\rho_{t}$ of $M_{d}$ is irreducible, this is impossible.

Let $X=N / \Delta$ be a compact nilmanifold, $H$ a subgroup of the automorphism group $A u t(X)$ of $X, m$ the Haar measure on $X$. It is known (see [1]) that, if there is no $N$-equivariant torus factor $T$ of $X$ such that the projection of $H$ in $A u t(X)$ is virtually abelian, then the natural representation $\rho_{0}$ of $H$ in $\mathbb{L}_{0}^{2}(X)$ does not weakly contain the identity representation. It follows that if $A \subset H$ generates $H$ and $\mu \in M^{1}(A)$ is aperiodic, i.e $\operatorname{supp}(\mu)$ is not contained 
in a coset of a proper subgroup of $H$, then the corresponding Markov operator $Q_{0}=\rho_{0}(\mu)$ satisfies $r\left(Q_{0}\right)<1$. We recall that a finitely generated group $\Gamma$ is said to be virtually abelian if it contains a normal group $\mathbb{Z}^{r}$ such that the quotient group $\Gamma / \mathbb{Z}^{r}$ is finite. We consider the random walk on $X$ defined by $\mu$ and we denote by $x_{n}=a_{n} \cdots a_{1} x\left(a_{i} \in \operatorname{supp} \mu, x \in X\right)$ its trajectories, where $x$ is distributed according to $m$. Let $f$ be an $\mathbb{R}$-valued function on $X$ with $m(f)=0, m\left(f^{2}\right)<\infty$ and let us consider the Birkhoff sums $S_{n}(x, \omega)=\sum_{0}^{n-1} f\left(x_{k}\right)$ with respect to the product measure $\mathbb{P}_{m}=m \otimes \mathbb{P}$ on $X \times H^{\mathbb{N}}$. We denote by $\mathbb{E}_{m}$ the corresponding expectation symbol. Then Theorem 1.7 gives :

Theorem 3.8 With the above notations, assume that the group $H=\langle\operatorname{supp}(\mu)\rangle \subset$ Aut $X$ is ergodic on $X$, that $\mu$ is aperiodic on $H$ and there is no $N$-equivariant torus factor of $X$ such that the corresponding projection of $H$ is virtually abelian. Let $f \in \mathbb{L}_{0}^{2}(X)$ be such that the equation $e^{i t f(x)}=e^{i \theta}$ is not satisfied $m$ - a.e for any $(t, \theta) \in \mathbb{R}^{2} \backslash\{0\}$.

Then there exists $\sigma(\mu, f)>0$ such that for any $\varphi \in \mathbb{L}^{2}(X)$ any $\psi$ continuous with compact support on $\mathbb{R}$ :

$$
\lim _{n \rightarrow \infty} \sigma(\mu, f) \sqrt{2 \pi n} \mathbb{E}_{m}\left(\varphi\left(x_{n}\right) \psi\left(S_{n}\right)\right)=m(\varphi) \ell(\psi) .
$$

We observe that there are natural subgroups of automorphims of $X$ without any hyperbolic element which satisfy the above hypothesis (see [10]).

Proof of Theorem 3.6 As in the above example we use Theorem 1.7. The condition $r\left(Q_{0}\right)<1$ follows from [1]. Here we have $Q^{i t} \varphi(x)=\sum_{a \in A} \varphi(a x) e^{i t f(a x)} \mu(a)$. Hence the condition $r\left(Q^{i t}\right)<1$ for $t \neq 0$ from Proposition 3.4 will be satisfied if there is no $t \neq 0$, $\varphi_{t} \in \mathbb{L}^{2}(X), \theta \in \mathbb{R}$ such that, for any $a \in \operatorname{supp}(\mu)$ and $m-$ a.e :

$$
e^{i t f(a x)}=e^{i \theta} \frac{\varphi_{t}(a x)}{\varphi_{t}(x)}
$$

This equation implies $e^{i t f(a x)} \varphi_{t}^{-1}(a x)=e^{i \theta} \varphi_{t}^{-1}(x)$, hence $e^{i t f(a x)} \varphi_{t}^{-1}(a x)$ is independant of $a \in \operatorname{supp} \mu$. Since $(\operatorname{supp}(\mu))(\operatorname{supp}(\mu))^{-1}$ generates $H$ and $H$ is ergodic we get $e^{i t f(x)} \varphi_{t}^{-1}(x)=$ $c, m$-a.e with $|c|=1$. It follows $e^{i \theta} \varphi_{t}^{-1}(x)=c$, hence $\varphi_{t}(x)=c^{-1} e^{i \theta}, e^{i t f(x)}=e^{i \theta}, m-a . e$, which is excluded.

\section{Extreme value theory for affine random walks.}

We consider the affine group $H$ of the vector space $V=\mathbb{R}^{d}$, a probability $\lambda$ on $H$ with projection $\mu$ on $\widetilde{G}=G L(V)$. We denote by $\widehat{\mathbb{P}}$ the product measure $\lambda^{\otimes \mathbb{N}}$ on $\widehat{\Omega}=H^{\mathbb{N}}$, we consider the dynamical system $(\widehat{\Omega}, \widehat{\theta}, \widehat{\mathbb{P}})$ where $\widehat{\theta}$ is the shift on $\widehat{\Omega}$, and we study some limit theorems for the stochastic recursion $x_{n+1}=g_{n+1} x_{n}+b_{n+1}, x_{0}=x$ where $\left(g_{n}, b_{n}\right)$ are i.i.d random variables with law $\lambda$. Under hypothesis $(C)$ below there exists a unique $\lambda$-invariant probability $\rho$ on $V$. We recall below the "homogeneity at infinity" property of 
$\rho$ (see [31], [32]), first observed in [38] in a weak form, and we describe its consequences for extreme value theory of the process $\left(x_{n}\right)_{n \in \mathbb{N}}$. The proofs of homogeneity are given here in a restricted setting and are based on the properties of the operators $P_{\mu}^{s}$ already studied in section 2 . Here we consider also the case $V=\mathbb{R} ;$ then we say that a probability $\mu$ on $\mathbb{R}^{*}$ is arithmetic if the semigroup $[\operatorname{supp}(\mu)]$ is contained in a subgroup $\pm a^{\mathbb{Z}}$ of $\mathbb{R}^{*}(a>0)$. If $d \geq 1$, our main hypothesis will be the existence of $\rho$, condition (I-P) for $[\operatorname{supp}(\mu)]$ and the existence of $g, g^{\prime}$ in $[\operatorname{supp}(\mu)]$ with $r(g)<1, r\left(g^{\prime}\right)>1$. More precisely condition $(C)$ is the following.

a) The semigroup $[\operatorname{supp}(\lambda)]$ has no fixed point in $V$.

b) There exists $\alpha>0$ with $k(\alpha)=\lim _{n \rightarrow \infty}\left(\int|g|^{\alpha} d \mu^{n}(g)\right)^{1 / n}=1$.

c) For some $\delta>0$ we have : $\int\left(|g|^{\alpha} \gamma^{\delta}(g)+|b|^{\alpha+\delta}\right) d \lambda(h)<\infty$.

d) If $d>1$, $[\operatorname{supp}(\mu)]$ satisfies condition (I-P) and if $d=1$, $[\operatorname{supp}(\mu)]$ is non-arithmetic.

Condition $(C)$ implies that $\log k^{\prime}(0)=\lim _{n \rightarrow \infty} \frac{1}{n} \log \left|S_{n}\right|<0$. We observe that $x_{n}-S_{n} x$ has the same law as $\sum_{k=1}^{n} g_{1} \cdots g_{k-1} b_{k}$, i.e as the partial sums of the convergent series $R=$ $\sum_{1}^{\infty} g_{1} \cdots g_{k-1} b_{k}$. Since $\lim _{n \rightarrow \infty} S_{n} x=0$, we have the equation : $R(\omega)=g_{1} R(\widehat{\theta} \omega)+b_{1}$, hence the law $\rho$ of $R$ is the unique $\lambda$-invariant probability. We observe that the above formula for $R$ implies $\int|x|^{s} d \rho(x)<\infty$ for $s<\alpha$. Property $b$ ) of condition $(C)$ implies the unboundedness of $\operatorname{supp}(\rho)$. We denote by $\eta \rightarrow t . \eta$ the extension of the dilation $v \rightarrow t v$ to measures on $V$, by $\ell^{s}$ the measure $\frac{d t}{t^{s+1}}$ on $\mathbb{R}_{+}$, by $\widetilde{\Lambda}([\operatorname{supp}(\mu)])$ the inverse image in $\mathbb{S}(V)$ of the limit set $\Lambda([\operatorname{supp}(\mu)]) \subset \mathbb{P}(V)$ considered in section 2 . Then we have the following "homogeneity property of infinity" for $\rho$, which is basic for the whole development of extreme value theory (see [41]) :

Theorem 4.1 Under condition $(C)$, the $\lambda$-invariant probability $\rho$ satisfies :

$$
\lim _{t \rightarrow 0_{+}} t^{-\alpha}(t . \rho)=c \widetilde{\sigma}^{\alpha} \otimes \ell^{\alpha}
$$

where $c>0, \widetilde{\sigma}^{\alpha}$ is a probability measure on $\widetilde{\Lambda}([\operatorname{supp}(\mu)])$ and $\widetilde{\sigma}^{\alpha} \otimes \ell^{\alpha}$ is a $\mu$-harmonic measure supported by $\mathbb{R} \widetilde{\Lambda}([$ supp $\mu])$. If [supp $\mu]$ has no proper convex invariant cone then $\widetilde{\sigma}^{\alpha}$ is equal to $\widetilde{\nu}^{\alpha}$, the unique symmetric measure on $\mathbb{S}(V)$ with projection $\nu^{\alpha}$ on $\mathbb{P}(V)$. If not, $\widetilde{\sigma}^{\alpha}$ is a convex combination of the two extremal $P^{\alpha}$-invariant probabilities on $\mathbb{S}(V)$ with projection $\nu^{\alpha}$ on $\mathbb{P}(V)$.

We observe that, in various situations, "homogeneity at infinity" of solutions of the corresponding functional equations can be deduced from the above theorem. This is the case (see [8]) in the study of the multidimensional "smoothing process" considered by Durett-Liggett in the context of statistical mechanics.

Below, we assume condition $(C)$ and we will restrict ourselves to the case where $[\operatorname{supp}(\mu)]$ has no proper convex invariant cone. The proof of Theorem 4.1 in the general case is 
long (see [31]). Here, with $H_{v}^{+}=\{x \in V ;\langle x, v\rangle \geq 1\}(v \in V \backslash\{0\})$, we show only the convergence of $t^{-\alpha}(t . \rho)\left(H_{v}^{+}\right)$under a reinforced hypothesis, and we deduce the homogeneity at infinity of $\rho$ if $\alpha \notin \mathbb{N}$. The proof of the positivity of $c$ in the general case is based on the well known Kac's return lemma and the method of ladder indices expounded in [14] for the case of random walks on the line ; here we will give an analytic argument in case $d=1$ only. If $d \geq 2$ we observe that the above homogeneity of $\rho$ is stable under perturbation of $\lambda$ in the weak topology ; it follows that convergence to Fréchet's law for affine random walks is robust if $d \geq 2$. This is also the case for the convergence to stable laws associated with affine random walks on $\mathbb{R}^{d}$ (see [17]).

In the special case considered above, the operator $\widetilde{P}_{\mu}^{z}$ defined by $\widetilde{P}_{\mu}^{z} \varphi(x)=\int|g x|^{z} \varphi(g \cdot x) d \mu(g)$ $(z=s+i t, s \geq 0, t \in \mathbb{R})$ on the unit sphere $\mathbb{S}(V)$ has spectral properties similar to those of $P_{\mu}^{z}$ in section 3. In particular, there exists a unique $\widetilde{P}_{\mu}^{s}$-eigenmeasure (resp eigenfunction) on $\mathbb{S}(V): \widetilde{P}_{\mu}^{s} \widetilde{\nu}^{s}=k(s) \widetilde{\nu}^{s}, \widetilde{P}_{\mu}^{s} \tilde{e}^{s}=k(s) \widetilde{\nu}^{s}$ and $\widetilde{\nu}^{s}$ (resp $\tilde{e}^{s}$ ) is the symmetric lift of $\nu^{s}$ (resp $\left.e^{s}\right)$ to $\mathbb{S}(V)$. The proof of Theorem 4.1 is based on a lemma and a proposition as follows.

Lemma 4.2 There exists an open set $D \subset \mathbb{C}$ which contains the set $\{\operatorname{Re} z \in] 0, \alpha]\}$ such that $\left(I-\widetilde{P}_{\mu}^{z}\right)^{-1}$ is meromorphic in $D$ with a unique simple pole at $z=\alpha$. Furthermore : $\lim _{z \rightarrow \alpha}(\alpha-z)\left(I-\widetilde{P}^{z}\right)^{-1}=k^{\prime}(\alpha)^{-1}\left(\widetilde{\nu}^{\alpha} \otimes \tilde{e}^{\alpha}\right)$.

Proof : If $d=1$, the lemma follows from elementary arguments and non-arithmeticity of $[\operatorname{supp}(\mu)]$. We assume now $d>1$. From the analogue of Theorem 2.20 in case $\mathbb{S}(V)$ it follows that for some $\varepsilon>0$ and any $z$ with $|z-\alpha|<\varepsilon$, there exists a holomorphic function $k(z)$ such that $k(z)$ is a dominant simple eigenvalue of $\widetilde{P}_{\mu}^{z}$ with $k(z)=1+k(\alpha)(z-\alpha)+\circ(z-\alpha)$. Since $k^{\prime}(\alpha) \neq 0$, we have $k(z) \neq 1$ for $z \neq \alpha$ and $|z-\alpha|$ small. Also for $|z-\alpha|$ small we have in $H^{\varepsilon}(\mathbb{S}(V))$ the decomposition $\widetilde{P}_{\mu}^{z}=k(z) \widetilde{\nu}^{z} \otimes \widetilde{e}^{z}+U(z)$ where $\widetilde{\nu}^{z} \otimes \widetilde{e}^{z}$ is a projection on $\mathbb{C} \widetilde{e}^{z}, U(z)$ satisfies $U(z)\left(\widetilde{\nu}^{z} \otimes \widetilde{e}^{z}\right)=\left(\widetilde{\nu}^{z} \otimes \widetilde{e}^{z}\right) U(z)=0, r(U(z))<1$ and $\widetilde{\nu}^{z} \otimes \widetilde{e}^{z}, U(z)$ depend holomorphically on $z$. We write :

$$
I-\widetilde{P}_{\mu}^{z}=(1-k(z))\left(\widetilde{\nu}^{z} \otimes \widetilde{e}^{z}\right)+p^{z}(I-U(z)),
$$

where $p^{z}=I-\widetilde{\nu}^{z} \otimes \widetilde{e}^{z}$. It follows $\left(I-\widetilde{P}_{\mu}^{z}\right)^{-1}=(1-k(z))^{-1}\left(\widetilde{\nu}^{z} \otimes \widetilde{e}^{z}\right)+p^{z}(I-U(z))^{-1}$, hence $\lim _{z \rightarrow \alpha}(\alpha-z)\left(I-\widetilde{P}_{\mu}^{z}\right)^{-1}=k^{\prime}(\alpha)^{-1}\left(\widetilde{\nu}^{\alpha} \otimes \widetilde{e}^{\alpha}\right)$ and $\left(I-\widetilde{P}_{\mu}^{z}\right)^{-1}$ is meromorphic in a disk $D_{0}$ centered at $\alpha$ with radius $\varepsilon^{\prime} \leq \varepsilon$, with unique pole at $z=\alpha$. For $z=\alpha+i t$ with $|t| \geq \varepsilon^{\prime}$, from the last assertion in Theorem 2.20 we get that there exists a disk $D_{t}$ centered at $\alpha+i t$ such that $r\left(\widetilde{P}_{\mu}^{z}\right)<1$ for $z \in D_{t}$, hence $\left(I-\widetilde{P}_{\mu}^{z}\right)^{-1}$ is a bounded operator depending holomorphically on $z$ for $z \in D_{t}$. If $\left.\operatorname{Re} z \in\right] 0, \alpha\left[\right.$, then $r\left(\widetilde{P}_{\mu}^{z}\right) \leq r\left(\widetilde{P}_{\mu}^{s}\right)<1$, hence $I-\widetilde{P}_{\mu}^{z}$ is invertible and the function $z \rightarrow\left(I-\widetilde{P}_{\mu}^{z}\right)^{-1}$ is holomorphic in the domain Rez $\left.\in\right] 0, \alpha[$. Then the open set $\left(\underset{t}{\cup} D_{t}\right) \cup\{\operatorname{Re} z \in] 0, \alpha[\}$ with $t=0$ or $|t| \geq \varepsilon^{\prime}$ satisfies the conditions of the lemma and the formula for $\lim _{z \rightarrow \alpha}(\alpha-z)\left(I-\widetilde{P}_{\mu}^{z}\right)^{-1}$ is valid.

We note that $R=\sum_{1}^{\infty} g_{1} \cdots g_{k-1} b_{k}$ satisfies the equation : $R=g_{1} R \circ \theta+B$ with $B=b_{1}$ 
We denote for $v \in V^{*}$ and $\left.\operatorname{Rez}=s \in\right] 0, \alpha[$ :

$$
f_{z}(v)=\widehat{\mathbb{E}}\left(\langle R, v\rangle_{+}^{z}\right), d_{z}(v)=\widehat{\mathbb{E}}\left(\langle R, v\rangle_{+}^{z}-\langle R-B, v\rangle_{+}^{z}\right) \text {. Then we have the }
$$

Proposition 4.3 We have the convergence:

$$
\lim _{t \rightarrow 0_{+}} t^{-\alpha}(t . \rho)\left(H_{u}^{+}\right)=c\left(\widetilde{\nu}^{\alpha} \otimes \ell^{\alpha}\right)\left(H_{u}^{+}\right)=C(u)=\alpha^{-1} \lim _{s \rightarrow \alpha_{-}}(\alpha-s) f_{s}(u)
$$

where $|u|=1, c \geq 0$ and $\widetilde{\nu}^{\alpha}$ satisfies $\mu *\left(\widetilde{\nu}^{\alpha} \otimes \ell^{\alpha}\right)=\widetilde{\nu}^{\alpha} \otimes \ell^{\alpha}$.

We have $C(u)=\left(\alpha k^{\prime}(\alpha)\right)^{-1}{ }^{*} \widetilde{\nu}^{\alpha}\left(\bar{d}_{\alpha}\right){ }^{*} \widetilde{e}^{\alpha}(u)$ where $\bar{d}_{\alpha}$ is the restriction of $d_{\alpha}$ to $\mathbb{S}\left(V^{*}\right)$.

Proof: We write the above functional equation in the form $R-B=g_{1} R \circ \widehat{\theta}$, hence for $R e z=s \in] 0, \alpha\left[\right.$ we have $\langle R-B, v\rangle_{+}^{z}=\left\langle R \circ \widehat{\theta}, g_{1}^{*} v\right\rangle_{+}^{z},{ }^{*} P_{\mu} f_{z}=f_{z}^{1}$, with $f_{z}^{1}(v)=\widehat{\mathbb{E}}\langle R-B, v\rangle_{+}^{z}$, i.e $\left(I-{ }^{*} P_{\mu}\right) f_{z}=d_{z}$. By homogeneity of $f_{z}, d_{z}$ and writing $\bar{f}_{z}, \bar{d}_{z}$ for the restrictions of $f_{z}, d_{z}$ to $\mathbb{S}(V)$ we get :

$$
\left(I-{ }^{*} P_{\mu}^{z}\right) \bar{f}_{z}=\bar{d}_{z}
$$

The function $\bar{d}_{z}(u)$ is dominated by :

$$
\varepsilon^{\prime}(z) \widehat{\mathbb{E}}\left(|B|^{s}\right)+\varepsilon(z) \widehat{\mathbb{E}}\left(|B|\left(|B|+<R, u>_{+}\right)^{s-1}\right)
$$

where $\varepsilon(z)=|z| 1_{\left[1, s_{\infty}[\right.}(s), \varepsilon^{\prime}(z)=1_{[0,1]}(s)$.

Hence, using the Hölder inequality and the moment hypothesis, we get that for $u$ fixed the function $z \rightarrow \bar{d}_{z}(u)$ is holomorphic in the domain $\left.\operatorname{Re} z \in\right] 0, \alpha+\delta[$; on the other hand the above lemma shows the meromorphicity of $z \rightarrow\left(I-{ }^{*} P_{\mu}^{z}\right)^{-1}$ in an open set $D$ which contains $\{\operatorname{Re} z \in] 0, \alpha]\}$. The above estimation of $\bar{d}_{z}(u)$ shows that the same property is valid for $\bar{f}_{z}=\left(I-{ }^{*} \widetilde{P}_{\mu}^{z}\right)^{-1}\left(\bar{d}_{z}\right)$. If we denote by $\rho_{u}$ the law of $<R, u>_{+}$, we have $\bar{f}_{s}(u)=\int x^{s} d \rho_{u}(x)$, hence $\bar{f}_{s}(u)$ is the Mellin transform of the positive measure $\rho_{u}$. Then, since $\left(I-{ }^{*} \widetilde{P}_{\mu}^{z}\right)^{-1}$ has a simple pole at $z=\alpha$, we can apply Wiener-Ikehara theorem (see [54]) to $\bar{f}_{s}(u), \rho_{u}$ and obtain the tail of $\rho_{u}$ in the form : $\lim _{t \rightarrow \infty} t^{\alpha} \rho_{u}(t, \infty)=\lim _{s \rightarrow \alpha_{-}} \alpha^{-1}(\alpha-s) \bar{f}_{s}(u)$. Hence, using Lemma 4.2 :

$$
\left.\lim _{s \rightarrow \alpha_{-}}(\alpha-s) \bar{f}_{s}(u)=k^{\prime}(\alpha)\right)^{-1 *} \widetilde{\nu}^{\alpha}\left(\bar{d}_{\alpha}\right) * \widetilde{e}^{\alpha}(u) .
$$

Using the formula for ${ }^{*} \widetilde{e}^{\alpha}(u)$ given in Lemma 2.23, we get :

$$
\lim _{s \rightarrow \alpha_{-}}(\alpha-s) \bar{f}_{s}(u)=\alpha C(u)=\alpha C\left(\widetilde{\nu}^{\alpha} \otimes \ell^{\alpha}\right)\left(H_{u}^{+}\right)
$$

with $C \geq 0, C(u) \geq 0$ and $\mu *\left(\widetilde{\nu}^{\alpha} \otimes \ell^{\alpha}\right)=\widetilde{\nu}^{\alpha} \otimes \ell^{\alpha}$.

For $d=1$ and some additionnal hypothesis we show $\lim _{t \rightarrow \infty} t^{\alpha} \rho(t, \infty)>0$, using an analytic argument sketched in [27]. This argument uses a lemma of E. Landau (see [54]) which says the following : if the Mellin transform of a positive measure $\rho$ supported on $[0, \infty[$ can be extended holomorphically to a neighbourbood of $\alpha \in \mathbb{R}_{+}$, containing $[\alpha, \alpha+\varepsilon[$ then we have $\int_{0}^{\infty} x^{s} d \rho(x)<\infty$ if $s<\alpha+\varepsilon$. Then we have the : 
Proposition 4.4 Assume $d=1, \lambda$ satisfies condition $C$, has compact support and $\operatorname{supp}(\mu) \subset$ $\mathbb{R}_{+}$. Then if $\operatorname{supp}(\rho) \cap \mathbb{R}_{+}$is unbounded, then :

$$
\lim _{t \rightarrow \infty} t^{\alpha} \rho(t, \infty)=c_{+}>0
$$

Proof : We know that $R=\sum_{0}^{\infty} g_{1} \cdots g_{k} b_{k+1}$ satisfies the equation : $R-b_{1}=g_{1} R \circ \widehat{\theta}$. Since $g_{1}$ is positive here we get $\left(R-b_{1}\right)_{+}=g_{1} R_{+} \circ \widehat{\theta}$.

With $\left.f(z)=\widehat{\mathbb{E}}\left(R_{+}^{z}\right), \quad f^{1}(z)=\widehat{\mathbb{E}}\left(R-b_{1}\right)_{+}^{z}\right), d(z)=f(z)-f^{1}(z)$, we get for $s<\alpha$ :

$$
\left(E_{0}\right) \quad k(z) f(z)=f_{1}(z), \text { and }(E) \quad(1-k(z)) f(z)=d(z) .
$$

Here we assume $\operatorname{supp}(\mu) \subset] 0, K[\operatorname{supp}(\lambda) \subset] 0, K] \times[-B, B]$, for some positive $K$ and $B$, hence the functions $k(z)=\widehat{\mathbb{E}}\left(g_{1}^{z}\right), \widehat{\mathbb{E}}\left(\left|b_{1}\right|^{z}\right)$ are holomorphic for $R e z>0$. Using the mean value theorem we get $\left|R_{+}^{z}-\left(R-b_{1}\right)_{+}^{z}\right| \leq B^{s}+|z| B\left(R_{+}+B\right)^{s-1}$, hence $|d(z)|<\infty$ for $s<\alpha+1$. Also, since $k(\alpha)=1$ and $\mu$ is non arithmetic we have $|k(\alpha+i t)|<1$ for $t \neq 0$. Then equation $(E)$ implies that $f(z)$ extends meromorphically to an open set $U \supset\{0<\operatorname{Re} z \leq \alpha\}$, with possibly a unique simple pole at $z=\alpha$. Then, as in the proof of Proposition 4.3 we get $\lim _{t \rightarrow \infty} t^{\alpha} \rho(t, \infty)=\alpha^{-1} \lim _{s \rightarrow \alpha}(\alpha-s) f(s)=A$. If $A=0$, since $k(s)>1$ for $s>\alpha, f(z)$ is holomorphic in a neighbourhood of ]0, $\alpha+1$ [, hence the Landau Lemma quoted above implies that $f(z)$ is holomorphic for $0<\operatorname{Re} z<\alpha+1$ and $\int_{0}^{\infty} x^{s} d \rho(x)<\infty$ for $s<\alpha+1$. Since $k(s)>1$ for $s>\alpha$, the function $(1-k(z))^{-1}$ is holomorphic in a neighbourhood of $\mathbb{R}_{+}$. Then as above, the Landau Lemma and equation $(E)$ imply that the Mellin transform $f(z)$ is holomorphic for Rez $>0$, and $f(s)=\int_{0}^{\infty} x^{s} d \rho(x)<\infty$ for $s \geq 0$. Now equation $\left(E_{0}\right)$ gives for $s \geq 1$

$$
k^{1 / s}(s) f^{1 / s}(s)=\widehat{\mathbb{E}}\left(\left(R-b_{1}\right)_{+}^{s}\right)^{1 / s} \leq \widehat{\mathbb{E}}\left(R_{+}^{s}\right)^{1 / s}+B .
$$

Hence $\left(k^{1 / s}(s)-1\right) f^{1 / s}(s) \leq B$.

Since $1<\lim _{s \rightarrow \infty} k^{1 / s}(s)=K_{1} \leq K$, it follows : $\lim _{s \rightarrow \infty} f^{1 / s}(s) \leq B\left(K_{1}-1\right)^{-1}<\infty$. Hence we have : $\operatorname{supp}(\rho) \subset]-\infty, B\left(K_{1}-1\right)^{-1}$ ], which contradict the hypothesis $\operatorname{supp}(\rho) \cap \mathbb{R}_{+}$ unbounded.

Proof of Theorem 4.1 We consider only the case where $[\operatorname{supp}(\mu)]$ has no proper convex invariant cone and $\alpha \notin \mathbb{N}$. Then using Proposition 4.3, and the properties of Radon transforms of positive measures studied in [2], we get the homogeneity at infinity result for $\rho$ if $\alpha \notin \mathbb{N}$. For $\alpha \in \mathbb{N}$ we use radial Fourier analysis (see [31]).

Theorem 4.1 allows to describe the asymptotic behaviour of large values of $x_{n}$, following the framework of extreme value theory (see [40], [41]). We denote $M_{n}=\sup \left\{\left|x_{i}\right| ; 1 \leq\right.$ $i \leq n\}$. We recall that Fréchet's law with parameters $p, \alpha$ is the probability measure $\Phi_{\alpha, p}$ on $\mathbb{R}_{+}$given by $\Phi_{\alpha, p}(0, t)=e^{-p t^{-\alpha}}$. For i.i.d random variables $x_{k}$ with law $\nu$ such that $\lim _{t \rightarrow \infty} t^{\alpha} \nu(t, \infty)=c>0$, it was observed by M. Fréchet (see [41]) that the law of $n^{-1 / \alpha} M_{n}$ converges to $\Phi_{\alpha, c}$. For affine random walks it can be shown (see [31]) that Fréchet's law is 
still valid but with a parameter $0<p<c$, an inequality due to the clustering of extreme values, which corresponds to the mixing with speed properties of the weakly dependant sequence $x_{n}$ (see Lemma 4.7 below). For analogous results on different homogeneous spaces see [39].

Proposition 4.5 With the notations and hypothesis of Theorem 4.1, the law of $n^{-1 / \alpha} M_{n}$ converges to the Fréchet law $\Phi_{\alpha, p}$ with $p<c$.

As observed in [44] in the case of geodesic flow on the modular surface, the famous Sullivan's logarithm law (see [50]) is a simple consequence of such a result. Here we have the

Corollary 4.6 For any $v \in V, x_{0}=v$, we have the $\widehat{\mathbb{P}}$ - a.e convergence :

$$
\limsup _{n \rightarrow \infty} \frac{\log \left|x_{n}\right|}{\log n}=\frac{1}{\alpha}
$$

The proofs of extreme value properties for the sequence $x_{n}$ are based on the nice spectral properties of the family of operators $P^{f, t}$ defined below. Let $f$ be a Lipchitz function which compact support on $[0,1] \times V$. For $\chi>0$, and $\varphi \in C(V)$, we write $|\varphi|_{\chi}=\sup \frac{|\varphi(v)|}{(1+|v|) \chi}$. For $\varepsilon \in] 0,1\left[, \lambda \geq 0\right.$ with $\lambda+\varepsilon<\chi$ we write $[\varphi]_{\varepsilon, \lambda}=\sup \frac{|\varphi(x)-\varphi(y)|}{|x-y|^{\varepsilon}\left(1+|x|^{\lambda}\left(1+|y|^{\lambda}\right)\right.}$, we define the space $H^{\chi, \varepsilon, \lambda}$ by $|\varphi|_{\chi}+[\varphi]_{\varepsilon, \lambda}<\infty$ and we norm $H^{\chi, \varepsilon, \lambda}$ by : $\|\varphi\|=|\varphi|_{\chi}+[\varphi]_{\varepsilon, \lambda}$. Then $H^{\chi, \varepsilon, \lambda}$ endowed with this norm is a Banach space and for suitable values of $\chi, \varepsilon, \lambda$ the "Laplace operator" $P^{f, t}$ defined by $P^{f, t} \varphi(v)=\int e^{-f(t, h v)} \varphi(h v) d \lambda(h)$ acts continuously on it. The use of the spectral properties of operators $P^{f, t}$ allows one to prove multiple mixing properties of the sequence $x_{n}$, in particular the following Lemma 4.7 below.

We consider the Markov probability $\widehat{\mathbb{P}}_{\rho}$ on the product space $V^{\mathbb{N} \cup\{0\}}$, associated with the affine random walk defined by $\lambda$ and the invariant probability $\rho$. We denote $r_{n}=[n]^{s}$ with $0<s<\alpha$ and $f_{j, n}(v, \omega)=\exp \left(-f\left(n^{-1} j, n^{-1 / \alpha}<v, x_{j}>\right)(1 \leq j \leq n)\right.$.

Lemma 4.7 With $r_{n}, f_{j, n}$ as above, $k_{n}=\left[n r_{n}^{-1}\right]$ we have :

$$
\lim _{n \rightarrow \infty}\left|\widehat{\mathbb{P}}_{\rho}\left(\prod_{j=1}^{n} f_{j, n}\right)-\prod_{k=1}^{k_{n}} \widehat{\mathbb{P}}_{\rho}\left(\prod_{j=1+(k-1) r_{n}}^{k r_{n}} f_{j, n}\right)\right|=0
$$

For the use of complex versions of the operators $P^{f, t}$ in the proofs of limit theorems, in particular for spectral gap properties and convergence to stable laws of Birkhoff sums $\sum_{j=1}^{n} x_{i}$, after normalisation, see $([17],[28])$. 


\section{References}

[1] Bekka B. and Guivarc'h Y. : On the spectral theory of groups of affine transformations on compact nilmanifolds. arXiv : 1106. 2623.

[2] Boman J. and Lindskog F. : Support theorems for the Radon transform and CramerWold theorems. J. Theoret. Probab. 22 (3), 683-710 (2009).

[3] Bourgain J. and Gamburd A. : Spectral gaps in SU(d). C.R.A.S ser I, 348, 609-611 (2010).

[4] Bougerol P. and Lacroix J. : "Products of random matrices with applications to Schrödinger operators" Progress in Probability and Statistics. Birkhauser, Boston Inc, Boston MA (1985).

[5] Bowen R. : Equilibrium states and the ergodic theory of Anosov diffeomorphisms. Springer Lecture Notes $\mathrm{n}^{\circ} 470$ (1975).

[6] Breiman L. : 'Probability". Addison-Wesley (1968).

[7] Broise A., Dal'bo F., Peigné M. : Etudes spectrales d'opérateurs de transfert et applications. Astérisque 238, S.M.F. (1996).

[8] Buraczewski D., Damek E., Guivarc'h Y., Mentemeier S. : On multidimensional Mandelbrot cascades. arXiv : 1109-1845 v2 (2014).

[9] Cantat S. and Le Borgne S. : Théorème limite central pour les endomorphismes holomorphes et les correspondances modulaires. Int. Math. Res. Not. 56, 3479-3510. (2005).

[10] Conze J.P. and Guivarc'h Y. : Ergodicity of group actions and spectral gap. Application to random walks and Markov shifts. Discrete and Continuous Dynamical Systems, Vol 33, n9 (2013).

[11] Dinh T.C., Sibony N. : Dynamics in several complex variables : Endomorphisms of projective spaces and polynomial-like mappings. In "Holomorphic Dynamical Systems" Springer Lecture Notes 1998, 165-289 (2008).

[12] Doeblin W., Fortet R. : Sur les chaînes à liaisons complètes. Bulletin SMF t 65, 132-148 (1937).

[13] Dolgopyat D., Goldsheid I. : Quenched limit theorems for nearest neighbour random walks in 1D random environment. Comm. Math. Phys. 315, 1, 247-277 (2012).

[14] Feller W. : "An Introduction to Probability Theory and its Applications" vol II, 2nd Ed. Wiley New York (1971). 
[15] Furman A. and Shalom Y. : Sharp ergodic theorems for group actions and strong ergodicity. Ergodic Theory Dynam. Systems, 19, 1037-1061 (1999).

[16] Furstenberg H. : Boundary theory and stochastic processes on homogeneous spaces. Proc. Symp. Pure Math. 36, 193-229 (1972).

[17] Gao Z. and Guivarc'h Y., Le Page E. : Spectral gap properties and convergence to stable laws for affine random walks. arXiv 1108-3146 (2011).

[18] Goldsheid I. and Margulis G.A. : Lyapunov exponents of a product of random matrices. Russian Math. Surveys 44 : 5, 11-71 (1989).

[19] Goldsheid I. and Guivarc'h Y. : Zariski closure and the dimension of the Gaussian law of the product of random matrices, I. Probab. Theory Related Fields 105, 109-142 (1996).

[20] Gouézel S. : Berry-Essen theorem and local limit theorem for non uniformly expanding maps. Ann. Inst. H. Poincaré 41, 997-1024 (2005).

[21] Guivarc'h Y. : Equirépartition dans les espaces homogènes. In "Théorie Ergodique, Actes Journées Ergodiques, Rennes 1973/1974". Lecture Notes in Math. vol 532, 131143, Springer-Verlag Berlin(1976).

[22] Guivarc'h Y. : Quelques propriétés asymptotiques des produits de matrices aléatoires. Eight Saint-Flour Probability summer School 1978 p 177-250 Lecture Notes in Math 774, Springer, Berlin(1980);

[23] Guivarc'h Y. : Applications d'un théorème limite local à la transience et à la récurrence de marches de Markov. Springer Lecture Notes n¹096, 301-332 (1983).

[24] Guivarc'h Y. and Raugi A. : Products of random matrices and convergence theorems. Contemp. Math., 59, 31-54 (1986).

[25] Guivarc'h Y. and Hardy J. : Théorèmes limites pour une classe de chaines de Markov et applications aux difféomorphismes d'Anosov. Ann. Inst. H. Poincaré Probab. Stat. 24 (1), 73-98 (1988).

[26] Guivarc'h Y. : Limit theorems for random walks and products of random matrices. "Probability measures on groups : recent directions and trends", 255-330 Tata Inst. Fund. Res., Mumbai (2006).

[27] Guivarc'h Y. : Heavy tail properties of multidimensional stochastic recursions. "Dynamics and Stochastics : Festchrift in honor of M. S. Keane", vol. 48 of IMS Lecture Notes Monogr. Ser. Inst. Math. Statist. Beachwood, OH, 85-99 (2006). 
[28] Guivarc'h Y. and Le Page E. : On spectral properties of a family of transfer operators and convergence to stable laws for affine random walks. Ergodic Theory Dynam. Systems 28, 423-446 (2008).

[29] Guivarc'h Y. : On the Spectrum of a Large Subgroup of a Semisimple Group. J.Modern Dynamics $2 \mathrm{n}^{\circ} 1,15-42$ (2008).

[30] Guivarc'h Y. : On contraction properties for products of Markov driven random matrices. Journal of Math. Physics, Analysis, Geometry vol 4 n$^{\circ} 4$, 457-489 (2008).

[31] Guivarc'h Y. and Le Page E. : Spectral gap properties and asymptotics of stationary measures for affine random walks. arXiv : 1204.6004 v4 (2014).

[32] Guivarc'h Y., Le Page E. : Homogeneity at infinity of stationary solutions of Multivariate Affine Stochastic Recursions. In "Random Matrices and Iterated Random functions". Alsmeyer G. - Löwe M. Eds. Springer Proceedings in Mathematics and Statistics 53, pp 119-135 (2013).

[33] Guivarc'h Y., Le Page E. : Asymptotique des valeurs extrêmes pour les marches aléatoires affines. C.R.A.S, ser I 351, 703-705 (2013).

[34] Hennion H. : Sur un théorème spectral et son application aux noyaux Lipchitziens. Proc. Am. Math. Soc 118, 2, 627-634 (1993).

[35] Ionescu Tulcea C.T. and Marinescu G. : Théorie ergodique pour des classes d'opérations non complètement continues. Ann. Math. 52, 140-147 (1950).

[36] Kazhdan D. A. : Uniform distribution on the plane, (Russian). Trudy Moskov. Mat. Ob., 14, 299-305 (1965).

[37] Keller G. : Un théorème de la limite centrale pour une classe de transformations monotones par morceaux. CRAS ser I 291, 2, 155-158 (1980).

[38] Kesten H. : Random difference equations and renewal theory for products of random matrices. Acta Math. 131, 207-248 (1973).

[39] Kirseböm M.S. : Extreme value theory for random walks on homogeneous spaces. arXiv : 1304.1006. (2013).

[40] Kluppelberg C., Pergamentchikov S. : Extremal behaviour of models with multivariate random recurrence representation. Stoch. Process. Appl., 117 (2007).

[41] Leadbetter M.R., Lindgren G., Rootzen H. : "Extremes and Related Properties of Random Sequences and Processes". Springer, Berlin, 73-98 (1983). 
[42] Le Page É. : Théorèmes limites pour les produits de matrices aléatoires. In : "Probability measures on groups" p 258-303, Lectures Notes in Math. 928, Springer (1982).

[43] Letchikov A.V. : Products of unimodular independant random matrices. Russian Math. Surveys 51 (1), 49-96 (1996).

[44] Pollicott M. : Limiting distributions for geodesic excursions on the modular surface. Contemp. Math. 469, 117-185 (2008).

[45] Rosenblatt M. : "Markov processes, Structure and Asymptotic behaviour". SpringerVerlag. Berlin Heidelberg New York. (1971).

[46] Rousseau-Égèle J. : Un théorème de la limite centrale pour une classe de transformations dilatantes. Annals of Proba, $11 \mathrm{n}^{\circ} 3,772-788$ (1983).

[47] Schmidt K. : Asymptotically invariant sequences and action of $S L(2, \mathbb{Z})$ on the 2sphere. Israel J. Math., 37, 193-208 (1980).

[48] Sinai Ya. G. : Gibbs measures in ergodic theory. Russian Maths Surveys n 4 (166) 21-64 (1972).

[49] Spitzer F. : "Principles of random walk". Springer. New York, Berlin (1976).

[50] Sullivan D. : Disjoint spheres, Approximation by imaginary quadratic numbers and the logarithm law for geodesics, Acta Math. 149, 215-237 (2008).

[51] Varjú P.P. : Random walks in Euclidean spaces. arXiv : 1205.3399.

[52] Vircer A.-D. : Matrix and operator random products Teor. Verayatnost. i Primenen $24 \mathrm{n}^{\circ} 2,361-370(1979)$.

[53] Vorobets Y. : Action of Finitely Generated Groups and Semigroups on the Plane by Means of Isometries. Mathematical notes vol 75, 4, 489-512 (2004).

[54] Widder D.V. : "The Laplace transform". Princeton University Press. New Jersey (1946). 